\title{
Classical Path Methods in Line Broadening. I. The Classical Path Approximation
}

\author{
Earl W. Smith, ${ }^{*}$ C. R. Vidal, ${ }^{*}$ and J. Cooper** \\ Institute for Basic Standards, National Bureau of Standards \\ Boulder, Colorado 80302
}

(February 24, 1969)

\begin{abstract}
ine classical path approximation is reviewed in a manner which permits definitive statements concerning its region of validity.
\end{abstract}

Key words: Classical path methods; line broadening theory.

\section{Introduction}

The classical path approximation which is used in most line broadening theories, as well as many other atomic and molecular problems, is not always clearly understood because it is based on rather heuristic arguments. Previous derivations have relied more on physical insight than mathematical analysis hence such features as the neglect of "back reaction" and the assumption that the density matrix is "diagonal in wave packet states" are not given to concise mathematical statements.

In this paper, statistical methods are used in deriving a precise mathematical expression for the "thermal average." The approximations used will be given as mathematical statements which are easily understood both physically and mathematically. It is hoped that this approach will be of some pedagogical value in understanding the foundations of the usual classical path methods.

In the following paper [1] ${ }^{1}$, henceforth referred to as II, this approach will be extended to show how the usual binary collision theories are obtained, and a systematic method for improving these theories is outlined.

\section{Construction of a Mathematical Model}

The line broadening problem for gases (or plasmas) is basically a study of the distribution of spectral radiaF tion emitted or absorbed by the gas. The emission (or

*Radio Standards Physics Division, Plasma Physics, National Bureau of Standards Laboratories, Boulder, Colo. 80302

** Joint Institute for Laboratory Astrophysics and Department of Physics and Astrophysics, University of Colorado, Boulder, Colo. 80302.

1 Figures in brackets indicate the literature references at the end of this paper. absorption) is usually produced by a small number of particles, and the spectral lines are shifted and broadened by the interaction between these radiators and the remainder of the particles in the gas. Since the number of radiators is usually a very small fraction of the total number of particles in the gas, the average distance between radiators will be large enough that any direct interactions between them are negligible. We will therefore regard the gas as being divided into a large number of cells in such a way that each cell contains one radiator and a large number of perturbing particles. We will assume that the particles at the border between adjacent cells are effectively at infinity with respect to the radiators; that is, the interaction between a radiator and a perturber at the edge of a cell is negligibly small. The distribution of particles at the border between cells will then be uniform (i.e., not altered by the presence of the radiators) and we may neglect all interactions between cells provided that we maintain this uniform distribution near the edges of the cells.

We have now represented the gas by a collection of noninteracting cells. Each cell may be described by a Hamiltonian of the form

$$
H=H_{a}+H_{p}+V,
$$

where $H_{a}$ is an unperturbed Hamiltonian for the radiator, $H_{p}$ is an unperturbed Hamiltonian for the remaining particles in the cell (henceforth called the perturbers), and $V$ represents the interaction between the radiator and these perturbers.

The probability per unit time that a cell, initially in the state $|m\rangle$, will make a spontaneous dipole transition to another state, $|n\rangle$, is [2]

$$
P_{m n}=\left(4 \omega_{m n}^{3} / 3 \hbar c^{3}\right)|<m| \mathbf{d}|n>|^{2}
$$


where $\mid m>$ and $\mid n>$ are $H$ eigenstates belonging to the eigenvalues $E_{m}$ and $E_{n}, \omega_{m n}$ is the frequency difference $\left(E_{m}-E_{n}\right) / \hbar$, and $\mathbf{d}$ is the dipole moment of the cell. Since we are interested only in line spectra, we may exclude the continuum radiation produced by the perturbers (bremsstrahlung, etc.) by excluding the dipole moment of the perturbers. That is, $\mathbf{d}$ will be the dipole moment operator for the radiator, thus ignoring all transitions which do not involve a change in the dipole moment of the radiator. The power radiated when a cell makes a dipole transition from $\mid m>$ to $\mid n>$ is just $\hbar \omega_{m n} P_{m n}$, and the contribution this makes to the total power spectrum emitted by the gas is $\hbar \omega_{m n} P_{m n} \delta\left(\omega-\omega_{m n}\right)$ or

$$
P(\omega)=\left(4 \omega^{4} / 3 c^{3}\right) \delta\left(\omega-\omega_{m n}\right)|<m| \mathbf{d}|n>|^{2}
$$

if we use delta functions as natural lines. If one wishes to use very narrow Lorentzian natural lines [3], it is necessary only to use a complex frequency variable in place of $\omega$; this is of little practical importance although it is a mathematical convenience for some problems. In $P(\omega)$, negative values of $\omega$ (or $\left.\omega_{m n}\right)$ represent absorptive transitions.

The total power spectrum emitted by the gas is obtained by adding the contributions, $P(\omega)$, from each of the cells. Since all cells have the same spectrum of accessible states ( $H$ eigenstates), this will turn out to be a weighted sum of eq (3) over all possible initial and final states. If the total number of cells (total number of radiators in the gas) is $N_{R}$ and if the probability of finding a cell (one radiator plus its perturbers) in the initial state $\mid m>$ is $\rho_{m}$, then the number of cells initially in the state $\mid m>$ is $N_{R} \rho_{m}$. Since $P_{m n}$, given by eq (2), is the probability that a cell initially in the state $|m\rangle$ will make a dipole transition to the state $|n\rangle$, any given transition $|m>\rightarrow| n\rangle$ will occur in $N_{R} \rho_{m} P_{m n}$ of the cells in the gas. The power radiated by this group of cells is $\hbar \omega_{m n} N_{R} \rho_{m} P_{m n}$ and their contribution to the total power spectrum is $\hbar \omega_{m n} N_{R} \rho_{m} P_{m n} \delta\left(\omega-\omega_{m n}\right)$. We obtain the total power spectrum for the gas by summing this expression over all possible $|m>\rightarrow| n\rangle$ transitions. The total power radiated per unit frequency interval is thus given by

$$
P_{T}(\omega)=\left(4 N_{R} \omega^{4} / 3 c^{3}\right) \Sigma_{m n} \delta\left(\omega-\omega_{m n}\right)|<m| \mathbf{d}|n>|^{2} \rho_{m} .
$$

This expression is equivalent to summing eq (3) over all $|m>\rightarrow| n>$ transitions, weighting each term by $N_{R} \rho_{m}$, the number of cells initially in the state $m>$.

In most line broadening problems one is interested only in a small range of frequencies which pertains to a single broadened line. In such a case the factor $\omega^{4}$ in eq (4) does not change appreciably, and most of the frequency dependence of $P(\omega)$ is contained in the sum over $\delta\left(\omega-\omega_{m n}\right)$. We thus define a line shape function, $I(\omega)$, by

$$
P(\omega)=\left(4 N_{R} \omega^{4} / 3 c^{3}\right) I(\omega)
$$

$$
I(\omega)=\Sigma_{m n} \delta\left(\omega-\omega_{m n}\right)|<m| \mathbf{d}|n>|{ }^{2} \rho_{m} .
$$

The evaluation of $I(\omega)$ is the central problem of line broadening theories. Since eq (6) is not multiplied by $N_{R}$, we see that it is necessary to consider only a single cell (with appropriate weighting of the initial states) when evaluating $I(\omega)$. For this reason, most line broadening theories start out with a mathematical model which contains a single radiator immersed in a gas of perturbing particles. We have included this section merely to justify the use of such a model, and in all following sections we will refer to this model rather than the gas as a whole.

\section{The Weak Coupling Approximation}

Using the results of the previous section, it can easily be shown $[4,5,6]$ that the line shape for spectral radiation is given by

$$
\begin{aligned}
I(\omega) & =(2 \pi)^{-1} \int_{-\infty}^{\infty} e^{i \omega t} C(t) d t \\
& =\pi^{-1} \operatorname{Re} \int_{0}^{\infty} e^{i \omega t} C(t) d t
\end{aligned}
$$

where $C(t)$ is a correlation function which has the property $C^{*}(t)=C(-t)$. For dipole radiation, $C(t)$ is an autocorrelation function of the dipole operator $\mathbf{d}$ for the radiator. This function may be given by

$$
C(t)=\operatorname{Tr}\left\{\mathbf{d} \cdot T^{\dagger}(t) \mathbf{d} T(t) \rho\right\}
$$

where $T(t)$ and $\rho$ are the time development operator [7] and density matrix for the system (a radiator and its perturbers) and the trace is over all states of the system.

The autocorrelation function $C(t)$ may be given various physical interpretations. Some of these interpretations are discussed in detail in section 7 .

Following Fano $[8,9]$, we regard the system as being composed of two weakly coupling subsystems; subsystem " $a$ " is the radiator (e.g., an atom, molecule, etc.) while subsystem " $p$ " is the gas of perturbers. The Hamiltonian, $H$, for the system has been given in eq (1) by $\left(H_{a}+H_{p}+V\right)$ where $H_{a}$ and $H_{p}$ are Hamiltonians describing the radiator and perturber subsystems and $V$ represents a coupling interaction between these subsystems [10].

We assume that the radiator and the gas of perturbers are statistically independent; that is, the state of the perturbers does not depend explicitly on the state of the radiator, and vice versa. This is the neglect of "back reaction" in the density matrix. A mathematical statement of this approximation has been given by Fano [9] in terms of the density matrix:

$$
\rho=\rho^{(a)} \rho^{(p)},
$$

where $\rho^{(a)}$ and $\rho^{(p)}$ depend only on radiator and perturber variables respectively. As a first approximation we assume that each subsystem is in equilibrium: 


$$
\begin{aligned}
& \rho^{(a)}=\rho^{(a)}\left(H_{a}\right) \\
& \rho^{(p)}=\rho^{(p)}\left(H_{p}\right) .
\end{aligned}
$$

For a canonical ensemble, $\rho \propto \exp \{-H / k T\}$; this requires that $\exp \{-V \mid k T\}$ be replaced by unity leaving $\rho \propto \exp \left\{-\left(H_{a}+H_{p}\right) / k T\right\}$ which has the form required by eqs (9) and (10). The interpretation of this approximation is a matter of some importance and it will be discussed in detail in section 8 .

The assumption of statistical independence, eq (9), permits one to write the correlation function, eq (8), in the form

$$
\begin{aligned}
C(t) & =\operatorname{Tr}_{a}\left\{\mathbf{d} \cdot \operatorname{Tr}_{p}\left\{T^{\dagger}(t) \mathbf{d} T(t) \rho^{(p)}\right\} \rho^{(a)}\right\} \\
& =\operatorname{Tr}_{a}\left\{\mathbf{d} \cdot<T^{\dagger}(t) \mathbf{d} T(t)>\rho^{(a)}\right\}
\end{aligned}
$$

where the subscripts on the trace operations denote a trace over states of the indicated subsystem [9]. The bracket $\langle\ldots\rangle$ represents an ensemble average over the perturber subsystem. This average takes the place of the usual "thermal average."

\section{The Perturber Ensemble}

We have assumed that the radiator and the perturber subsystems are statistically independent. The density operator, $\rho^{(p)}$, for the perturber subsystem will therefore be the same as the density operator for an isolated gas of $N$ particles in a volume $\mathscr{V}$ (the volume of a cell) maintained at a constant temperature $T$. Given the restrictions $(N, \mathscr{V}, T)$, there are many possible configurations of positions, $\mathbf{x}=\left(\mathbf{x}_{1}, \mathbf{x}_{2} \ldots\right.$, $\left.\mathbf{x}_{N}\right)$, and velocities, $\mathbf{v}=\left(\mathbf{v}_{1}, \mathbf{v}_{2}, \ldots, \mathbf{v}_{N}\right)$, for the particles which are consistent with these macroscopic constraints. Speaking quantum mechanically, we say that there are many different state functions, $\varphi(\mathbf{x}, t)$, which could describe such an isolated gas. We may index each of these possibilities with a superscript $(i)$, and we imagine a collection of perturber gases, called an ensemble [11]. Each $\varphi^{(i)}(\mathbf{x}, t)$ must be a solution of the Schrödinger equation,

$$
i \hbar \frac{\partial}{\partial t} \varphi^{(i)}(\mathbf{x}, t)=H_{p} \varphi^{(i)}(\mathbf{x}, t),
$$

which is normalized to unity:

$$
\int\left|\varphi^{(i)}(\mathbf{x}, t)\right|^{2} d \mathbf{x}=1
$$

Due to its mathematical nature, the concept of an ensemble is not readily given to simple physical interpretations. However, in the following section it will be shown that, by neglecting the influence of the radiator on the state of the perturbers which surround it (i.e., "back reaction" effects in the wave functions), a physical interpretation of the ensemble is possible. In that case we may regard each of the cells discussed in section 2 as being a member of the ensemble (provided that there are enough cells); that is, each $\varphi^{(i)}$ will then represent one of the possible states for the perturbers in a cell and, for a given state of the radiator, the superscript $i$ may then be used to index the cells. It must be emphasized that in this section we are not representing the wave function for the perturbers in the cells by $\varphi^{(i)}$. At this point we are simply constructing an approximate density operator for these perturbers which is based on an isolated gas. In the following section we will use the $\varphi^{(i)}$ as basis functions in constructing wave functions for the perturbers in the cells, however, the treatment of the wave functions in the following section is distinct from the discussion of the statistics in this section.

For an isolated gas, energy would be a constant of the motion hence each $\varphi^{(i)}$ will be an eigenfunction of $H_{p}$ [13]. If we define a complete orthonormal set of $H_{p}$ eigenfunctions $\left\{f_{k}(\mathbf{x})\right\}$ by

$$
\begin{gathered}
H_{p} f_{k}(\mathbf{x})=E_{p}^{(k)} f_{k}(\mathbf{x}) \\
\int f_{k}^{*}(\mathbf{x}) f_{j}(\mathbf{x}) d \mathbf{x}=\delta_{k, j},
\end{gathered}
$$

then, from eqs (12) and (13), it is obvious that

$$
\varphi^{(i)}(\mathbf{x}, t)=f_{i}(\mathbf{x}) \exp \left(-i t E_{p}^{(i)} / \hbar\right) .
$$

Using the notation

$$
\begin{aligned}
f_{k}(\mathbf{x}) & =\langle\mathbf{x} \mid k\rangle \\
\varphi^{(i)}(\mathbf{x}, t) & =\left\langle\mathbf{x} \mid \varphi^{(i)} ; t\right\rangle
\end{aligned}
$$

Equation (16) may also be written in the form

$$
\left|\varphi^{(i)} ; t>=\right| i>\exp \left(-i t E_{p}^{(i)} \mid \hbar\right) .
$$

The probability of finding the gas of perturbers in a particular state $\varphi^{(i)}$ will be denoted by $q^{(i)}$, and the $q^{(i)}$ will be normalized so. that

$$
\Sigma_{i} q^{(i)}=1 .
$$

The density operator, $\rho^{(p)}$ is then defined [9] by a sum over all possible states $\varphi^{(i)}$, weighting each by its probability of occurrence $q^{(i)}$ :

$$
\begin{aligned}
\rho^{(p)} & =\sum_{i}\left|\varphi^{(i)} ; t>q^{(i)}<\varphi^{(i)} ; t\right| \\
& =\sum_{i}\left|i>q^{(i)}<i\right| .
\end{aligned}
$$

For the macroscopic constraints $N, \mathscr{V}$, and $T$, the statistical weights are given by

$$
q^{(i)}=\exp \left\{-E_{p}^{(i)} / k T\right\} / \sum_{i} \exp \left\{-E_{p}^{(i)} / k T\right\}
$$

where $E_{p}^{(i)}$ are the $H_{p}$ eigenvalues. Since the states $\varphi^{(i)}$ are $H_{p}$ eigenfunctions, they are also eigenfunctions of the operator $\exp \left(-H_{p} / k T\right) / \operatorname{Tr}_{p}\left\{\exp \left(-H_{p} / k T\right)\right\}$ 
having the eigenvalues $q^{(i)}$ as given by eq (21). Using this property and a closure relation for the $\varphi^{(i)}$ we may combine eqs (20) and (21) to obtain a more familiar form for $\rho^{(p)}$.

$$
\rho^{(p)}=\exp \left(-H_{p} / k T\right) / \operatorname{Tr}_{p}\left\{\exp \left(-H_{p} / k T\right)\right\} \cdot
$$

It is obvious, from this equation, that the perturber density matrix is diagonal between eigenstates of $H_{p}$. Since the states $\varphi^{(i)}(\mathbf{x}, t)$ are eigenfunctions of $H_{p}$, the density matrix will be diagonal between these states. In the following section it will be assumed that $\varphi^{(i)}(\mathbf{x}, t)$ may, under certain conditions, be viewed as a product of wave packets for each of the perturbers. We will then be able to state that the density matrix is "diagonal in wave packet states."

\section{Approximate Wave Functions}

In this section, approximations will be made concerning the form of the wave functions for the system (radiator and perturbers). We will derive the wave functions used by Baranger [4], retaining the superscript (i) notation of the previous section in order to clarify the influence of the perturbers in these wave functions.

The wave functions for the system must be solutions of the Schrödinger equation

$i \hbar \frac{\partial}{\partial t} \Psi(\mathbf{R}, \mathbf{x}, t)=\left(H_{a}+H_{p}+V\right) \Psi(\mathbf{R}, \mathbf{x}, t)$,

where $\mathbf{x}$ is a $3 N$-vector $\left(\mathbf{x}_{1}, \mathbf{x}_{2}, \ldots, \mathbf{x}_{N}\right)$, which denotes the positions of the $N$ perturbers, and $\mathbf{R}$ denotes the internal coordinates of the radiator (e.g., for an atom, $\mathbf{R}$ denotes the positions of the orbital electrons).

We wish to expand the wave functions $\Psi(\mathbf{R}$, $\mathbf{x}, t)$ in terms of some complete set of basis functions for the system. Since the functions $f_{k}(\mathbf{x})$, introduced in the previous section, form a complete set for the perturber subsystem and the $H_{a}$ eigenfunctions, which we shall call $g_{j}(\mathbf{R})$, form a complete set for the radiator subsystem, we may expand $\Psi(\mathbf{R}, \mathbf{x}, t)$ in the following manner:

$$
\Psi(\mathbf{R}, \mathbf{x}, t)=\sum_{j k} a_{j k}(t) g_{j}(\mathbf{R}) f_{k}(\mathbf{x}),
$$

where the $a_{j k}(t)$ are to be determined by using eq (23) and some boundary condition on $\Psi$.

Using eq (16) we may rewrite eq (24) in the form

$$
\Psi(\mathbf{R}, \mathbf{x}, t)=\sum_{k} \chi^{(k)}(\mathbf{R}, t) \varphi^{(k)}(\mathbf{x}, t)
$$

where the coefficients

$$
\chi^{(k)}(\mathbf{R}, t)=\sum_{j} a_{j k}(t) g_{j}(\mathbf{R}) \exp \left(i E_{p}^{(k)} t / \hbar\right)
$$

must be determined by requiring that $\Psi$ satisfy the Schrödinger equation, eq (23), as well as some boundary condition.
At some initial time, $t=0$, we may require that the perturber gas be represented by one of the wave functions $\varphi^{(i)}$ :

$$
\Psi(\mathbf{R}, \mathbf{x}, 0)=\chi^{(i)}(\mathbf{R}, 0) \varphi^{(i)}(\mathbf{x}, 0) .
$$

That is, at some instant in time, the coupling interaction $V$ will be small and the perturbers will behave like an isolated gas at that instant. Equation (27) constitutes the boundary condition for $\Psi$. Substituting eq (25) into the Schrödinger equation, eq (23), and using the orthogonality of the $\varphi^{(k)}$, we obtain a set of coupled equations for the coefficients $\chi^{(k)}$ :

$i \hbar \frac{\partial \chi^{(k)}}{\partial t}=\left[H_{a}+V^{(k)}(t)\right] \chi^{(k)}+\sum_{j \neq k} V^{(k, j)}(t) \chi^{(j)}$

where

$$
\begin{aligned}
& V^{(k)}(t)=\left\langle\varphi^{(k)} ; t|V| \varphi^{(k)} ; t\right\rangle \\
& V^{(j, k)}(t)=\left\langle\varphi^{(j)} ; t|V| \varphi^{(k)} ; t\right\rangle
\end{aligned}
$$

We will now assume that the state of the perturbers is not affected by the interaction $V$; that is,

$$
\left\langle\varphi^{(i)} ; t|V| \varphi^{(j)} ; t\right\rangle \ll\left\langle\varphi^{(i)} ; t|V| \varphi^{(i)} ; t\right\rangle .
$$

With this approximation the states $\varphi^{(i)}$ may be regarded as approximate $V$ eigenfunctions,

$$
V\left|\varphi^{(i)} ; t>=V^{(i)}\right| \varphi^{(i)} ; t>,
$$

as well as $H_{p}$ eigenfunctions. This relation provides a mathematical statement of the neglect of "back reaction" in the wave functions. Since the state of the perturbers is now unaffected by the presence of the atom, the coupling term in eq (28) vanishes and, using the boundary condition given in eq (27), we readily obtain the approximate wave function

$$
\Psi^{(i)}(\mathbf{R}, \mathbf{x}, t)=\chi^{(i)}(\mathbf{R}, t) \varphi^{(i)}(\mathbf{x}, t),
$$

where $\chi^{(i)}$ satisfies the equation

$$
i \hbar \frac{\partial}{\partial t} \chi^{(i)}=\left[H_{a}+V^{(i)}(t)\right] \chi^{(i)} .
$$

Comparing eqs (12), (29), (33), and (34) with eqs (16), (17), (21), and (22) of reference [4], we see that the approximate wave function $\Psi^{(i)}$ is identical with the approximate wave function introduced by Baranger. The superscript (i) now indicates the dependence of the total wave function, $\Psi^{(i)}$, on the various possible perturber configurations in a cell (as mentioned in the previous section). Although this dependence is implied by Baranger, it is not illustrated by such a parameter.

It is obvious that a function $\Psi^{(i)}(\mathbf{R}, \mathbf{x}, t)$ having the form given in eq (33) cannot rigorously satisfy the Schrödinger, equation, eq (23), because the latter 
is, in general, not separable equation. It would be possible to find solutions of the form $\chi^{(i)}(\mathbf{R}, \mathbf{x}, t) \varphi^{(i)}(\mathbf{x}$, t), but the "radiator wave function," $\chi^{(i)}$, would then depend explicitly on the perturber coordinates, $\mathbf{x}$, as well as $\mathbf{R}$ and $t$. By neglecting "back reaction," according to eq (32), we have obtained an approximate wave function of the form $\chi^{(i)} \varphi^{(i)}$ where $\chi^{(i)}$ does not depend explicitly on perturber coordinates. The superscript $(i)$ which appears on this "radiator wave function" indicates that $\chi^{(i)}$ does depend $i m$ plicitly on the state of the perturbers through the effective potential $V^{(i)}(t)$; that is, we get different functions $V^{(i)}$ for each of the possible states $\varphi^{(i)}$.

A general procedure for approximating the solution of the Schrödinger equation (23) by a function $\Psi^{(i)}$ having the form stated in eq (33) would be an iterative one. The first approximation uses $\varphi^{(i)}$ and $\chi^{(i)}$ as given by eqs (12) and (34). If we chose to, we could obtain the next approximation to $\varphi^{(i)}$ by substituting $\Psi^{(i)}=$ $\varphi^{(i)} \chi^{(i)}$ into the Schrödinger equation (23), using eq (34) to remove some of the $\chi^{(i)}$ terms. Multiplying by $\chi^{(i) *}$ and integrating over $d R$ then gives a differential equation for $\varphi^{(i)}$ which is similar in form to eq (34). That is, in addition to the $H_{p}$ which appears in eq (12) we would have a potential energy function which is averaged over $R$. The $\varphi^{(i)}$ solution of this new equation is presumably an improvement over the solution of eq (12) since the former contains some back reaction effects; however, the improved $\varphi^{(i)}$ would no longer be an $H_{p}$ eigenfunction. Since it is desirable that $\varphi^{(i)}$ be an $H_{p}$ eigenfunction and thus diagonalize $\rho^{(p)}$, we will use the first approximation to $\varphi^{(i)}$ (i.e., the solution of eq (12)). If back reaction effects are important, it is not sufficient to use this first approximation. If a higher order approximation is used, the density matrix, $\rho^{(p)}$, will not be diagonal, as noted by Griem [14]. [In sec. 8 it will be shown that when these back reaction effects become important, the classical path approximation is breaking down anyway.] In this case, it would be more convenient to use the $H_{p}$ eigenfunctions as a basis set in evaluating the trace over perturber coordinates (i.e., the thermal average as defined by eq (11)). The perturber density matrix would then be diagonal, but it would be necessary to make a fully quantum mechanical calculation of the time development operator (similar to that discussed by Baranger $[4,5]$ ). Such a procedure would not be a classical path calculation and we will not consider this case any further.

In keeping with the usual classical path treatment, we next assume that each wave function $\varphi^{(i)}(\mathbf{x}, t)$ may be expressed as a product of wave packet states $\varphi_{j}^{(i)}\left(\mathbf{x}_{j}, t\right)$ for each of the perturbers:

$$
\varphi^{(i)}(\mathbf{x}, t)=\prod_{j} \varphi_{j}^{(i)}\left(\mathbf{x}_{j}, t\right) .
$$

The wave packets, $\varphi_{j}^{(i)}$, are assumed to be sharply localized during the times of interest in line broadening. Although one does not usually think of energy eigenstates as being wave packets, it is nonetheless possible for a wave packet state to be an approximate eigenfunction of both $H_{p}$ and $V$ (as required by eq (32)) having eigenvalues $E_{p}^{(i)}$ and $V^{(i)}(t)$ which are classical functions of perturber coordinates. The validity of this approximation is discussed in section (8).

We now note that the influence of the perturbers which is implicit in $V^{(i)}(t)$ may be specified by giving the locations, $\mathbf{x}_{j}^{(i)}$, of the peaks of the packets $\varphi_{j}^{(i)}$, as well as their group velocities, $\mathbf{v}_{j}^{(i)}$. We thus complete the classical path picture by regarding the wave packets $\varphi_{j}^{(i)}$ as classical particles described by their positions

$\mathbf{x}^{(i)}=\left(\mathbf{x}_{1}^{(i)}, \mathbf{x}_{2}^{(i)}, \ldots, \mathbf{x}_{N}^{(i)}\right)$ and velocities

$$
\mathbf{v}^{(i)}=\left(\mathbf{v}_{1}^{(i)}, \mathbf{v}_{2}^{(i)}, \ldots, \mathbf{v}_{N}^{(i)}\right) .
$$

Since the wave packets $\varphi_{j}^{(i)}$ are assumed to be sharply localized, we may replace $V^{(i)}(t)$ by a classical potential function. That is,

$$
\int \varphi^{(i) *}(\mathbf{x}, t) V \varphi^{(i)}(\mathbf{x}, t) \mathbf{d} \mathbf{x}=V^{(i)}\left(\mathbf{R}, \mathbf{x}^{(i)}, \mathbf{v}^{(i)}, t\right)
$$

where $V^{(i)}\left(\mathbf{R}, \mathbf{x}^{(i)}, \mathbf{v}^{(i)}, t\right)$ is a classical potential function of the $3 N$-vectors $\mathbf{x}^{(i)}$ and $\mathbf{v}^{(i)}$, the operator $\mathbf{R}$, and the time $t$.

\section{The Thermal Average}

To obtain an expression for the "thermal average" in eq (11), we must first obtain an expression for the time development operator, $T(t)$, for the total system. Since we are approximating the wave function $\Psi(\mathbf{R}, \mathbf{x}, t)$ by $\Psi^{(i)}(\mathbf{R}, \mathbf{x}, t)$, we will need the corresponding time development operator $T^{(i)}(t)$ which is defined by

$$
\Psi^{(i)}(\mathbf{R}, \mathbf{x}, t)=T^{(i)}(t) \Psi^{(i)}(\mathbf{R}, \mathbf{x}, 0) .
$$

From eqs (33) and (34), we see that this operator may be written in the form

$$
T^{(i)}(t)=\exp \left\{-i t H_{p} / \hbar\right\} T_{a}^{(i)}(t)
$$

where $T_{a}^{(i)}(t)$ is the solution of

$$
i \hbar \frac{\partial}{\partial t} T_{a}^{(i)}(t)=\left[H_{a}+V^{(i)}\left(\mathbf{R}, \mathbf{x}^{(i)}, \mathbf{v}^{(i)}, t\right)\right] T_{a}^{(i)}(t)
$$

with the boundary conditions $T_{a}^{(i)}(0)=1$.

Since d (see eq (11)) is the dipole operator for the radiator, it will commute with the perturber Hamiltonian $H_{p}$ and we may write

$$
T^{(i)^{\dagger}}(t) \mathbf{d} T^{(i)}(t)=T_{a}^{(i)}{ }^{\dagger}(t) \mathbf{d} T_{a}^{(i)}(t) .
$$

Substituting this identity into the "thermal average" in eq (11), we obtain

$$
\begin{aligned}
\langle\ldots . .\rangle & =\operatorname{Tr}_{p}\left\{T_{a}^{(i)^{\dagger}}(t) \mathbf{d} T_{a}^{(i)}(t) \rho^{(p)}\right\} \\
& =\sum_{k \cdot k^{\prime}}\left\langle k\left|T_{a}^{(i)} \mathbf{d} T_{a}^{(i)}(t)\right| k^{\prime}\right\rangle\left\langle k^{\prime}\left|\rho^{(p)}\right| k\right\rangle,
\end{aligned}
$$


where $|k\rangle$ denotes an arbitrary basis vector for the perturber subsystem. Since $T_{a}^{(i)}(t)$ has no explicit dependence on perturber coordinates (i.e., it depends on perturbers implicitly through the coordinates $\mathbf{x}^{(i)}, \mathbf{v}^{(i)}$ of the wave packets $\left.\varphi^{(i)}\right)$, we have

$<k\left|T_{a}^{(i) \dagger}(t) \mathbf{d} T_{a}^{(i)}(t)\right| k^{\prime}>=\delta_{k, k^{\prime}} T_{a}^{(i) \dagger}(t) \mathbf{d} T_{a}^{(i)}(t) \cdot$

Substituting this identity into eq (41), we see that we need only the diagonal matrix elements of $\rho^{(p)}$ in the states $\mid k>$. Using the matrix elements of $\rho^{(p)}$ as given by eq (20) and noting the normalization condition of eq (16), we obtain

$$
<\ldots .>=\sum_{i} q^{(i)} T_{a}^{(i)}(t) \mathbf{d} T_{a}^{(i)}(t) .
$$

To complete our evaluation of the "thermal average," we must express this weighted average in a form which is amenable to practical calculations.

Since $q^{(i)}$ is a function of $E_{p}^{(i)}$, defined by eq (21), we may express $q^{(i)}$ as a function of $\mathbf{x}^{(i)}$ and $\mathbf{v}^{(i)}$ by using

$$
\begin{gathered}
<\varphi^{(i)} ; t\left|H_{p}\right| \varphi^{(i)} ; t>=E_{p}^{(i)}\left(\mathbf{x}^{(i)}, \mathbf{v}^{(i)}\right) \\
=\sum_{j} \frac{1}{2} m \mathbf{v}_{j}^{(i) 2}+V_{p}\left(\mathbf{x}^{(i)}\right)
\end{gathered}
$$

where $V_{p}$ denotes the interaction between perturbers. If the radiator is an ion in a plasma, we may add a Coulomb interaction, $V_{0}\left(\mathbf{x}^{(i)}\right)$, to $H_{p}[10]$. This expression is consistent with the wave packet interpretation of $\varphi^{(i)}(\mathbf{x}, t)$.

In the classical limit, the sum over $(i)$ is replaced by an integral over $\mathbf{x}^{(i)}$ and $\mathbf{v}^{(i)}$. We may thus write $q^{(i)}$ in the form

$$
q^{(i)}\left(\mathbf{x}^{(i)}, \mathbf{v}^{(i)}\right)=\exp \left\{-E_{p}^{(i)}\left(\mathbf{x}^{(i)}, \mathbf{v}^{(i)}\right) / k T\right\} / Q,
$$

where $Q$ is the classical partition function

$$
Q=\iint \exp \left\{-E_{p}^{(i)}\left(\mathbf{x}^{(i)}, \mathbf{v}^{(i)}\right) / k T\right\} d \mathbf{x}^{(i)} d \mathbf{v}^{(i)} .
$$

We may now write the "thermal average" in the form

$$
\begin{gathered}
<\ldots>=Q^{-1} \iint d \mathbf{x}^{(i)} d \mathbf{v}^{(i)} \exp \left\{-E_{p}^{(i)}\left(\mathbf{x}^{(i)}, \mathbf{v}^{(i)}\right) / k T\right\} \\
T_{a}^{(i)+(}\left(\mathbf{x}^{(i)}, \mathbf{v}^{(i)}, t\right) \mathbf{d} T_{a}^{(i)}\left(\mathbf{x}^{(i)}, \mathbf{v}^{(i)}, t\right)
\end{gathered}
$$

where the time development operator $T_{a}^{(i)}\left(\mathbf{x}^{(i)}, \mathbf{v}^{(i)}, t\right)$ is obtained by solving eq (39) with the classical potential $V^{(i)}\left(\mathbf{R}, \mathbf{x}^{(i)}, \mathbf{v}^{(i)}, t\right)$. This expression will be reduced to the more familiar averages over collision variables (velocity, impact parameter, etc.) in II.

\section{The Autocorrelation Function}

\subsection{The Influence of the Density Matrix and the Wave Functions}

Before discussing the validity of the approximations concerning the density matrix and the wave functions (sec. 8), it is useful to first consider the roles they play in determining the line shape. Since the line shape has been given as a Fourier transform of the autocorrelation function, eq (7), it is convenient to study this function as well.

The autocorrelation function was defined in eq (8) by $C(t)=\operatorname{Tr}\{\mathbf{d} \cdot \mathbf{d}(t) \rho\}$ where $\quad \mathbf{d}(t)=T^{\dagger}(t) \mathbf{d} T(t)$ and $\rho$ is an equilibrium density matrix for a radiator and its perturbers. Since both $\rho$ and $T(t)$ are functions of $H$ (see eq (1)), they will commute at all times (i.e., $\rho$ is stationary) and we may write

$$
\operatorname{Tr}\{\mathbf{d} \cdot \mathbf{d}(t) \rho\}=\operatorname{Tr}\left\{\mathbf{d}\left(t_{0}\right) \cdot \mathbf{d}\left(t+t_{0}\right) \rho\right\}
$$

for any time $t_{0}$. This relation is characteristic of a stationary random process and it indicates that when we speak of an initial time, we are referring to any arbitrarily chosen instant, not some time infinitely far in the past. In writing $C(t)=\operatorname{Tr}\{\mathbf{d} \cdot \mathbf{d}(t) \rho\}$ we have arbitrarily chosen $t=0$ as our initial time. Autocorrelation functions [15] such as $C(t)$ fall to zero as $t$ increases; this represents the loss of correlation between the dipole moment at the time $t=0$ and its value at some later time.

We may regard the trace, in the defining relation for $C(t)$, as a sum over all possible initial configurations of perturbers and initial states of the radiator; each term in this sum is weighted by the matrix element of $\rho$ which gives the probability of finding the corresponding initial state. We may also interpret this trace as a sum over cells, with each initial state corresponding to a given radiator-perturber configuration as discussed in section 2. For each initial state there will be a dipole moment, $\mathbf{d}$, and at some time $t$ later this moment will have changed to $\mathbf{d}(t)$. Once the initial state has been specified, the temporal change in $\mathbf{d}$ is determined solely by the time development operator $T(t)$, and is not influenced by the statistics (density matrix) in any way.

We may now think of the line shape as being obtained by taking the Fourier transform of the $\mathbf{d} \cdot \mathbf{d}(t)$ contribution from each initial state, and then summing these Fourier transforms, weighting each term by the appropriate density matrix element (the probability of occurrence of a given configuration in a cell). It is thus apparent that the density matrix can influence the line shape only by its weighting of each $\mathbf{d} \cdot \mathbf{d}(t)$ contribution; the wave functions influence $I(\omega)$ only by their effect on the calculation of each $\mathbf{d} \cdot \mathbf{d}(t)$ term. The influence of the density matrix is quite distinct from the influence of the wave functions, and similarly, approximations relevant to the statistics (density matrix) are completely independent of the approximations relevant to the dynamics (time development operator or wave functions).

\subsection{Classical Correspondence}

\section{a. Classical Theories}

In discussing any quantum mechanical theory it is frequently beneficial to have a classical correspondence available as an aid to understanding the physical 
properties underlying the mathematical description. In a classical path description of line broadening, the perturbers are regarded as classical particles, and there is little difficulty in understanding the physical properties of this subsystem. The radiator is treated quantum mechanically however, and it is useful to develop its classical analog to clarify the influence of collisions on the spectral radiation.

In classical line broadening theories one considers a classical oscillating dipole which emits a continuous wave train (referred to as the light train). The light train is described by the classical dipole moment $d(t)$, and the line shape is given by a Fourier transform of the dipole autocorrelation function $C(t)=\langle d d(t)\rangle$ just as in eq $(7)$; in this case $\langle\ldots\rangle$ denotes a classical average over collisions. In the Lorentz interruption theory (see p. 571 of ref. [16]), it is assumed that a collision terminates the unperturbed light train $d(t)=d \exp \left(-i \omega_{0} t\right)$ where $\omega_{0}$ is the unperturbed frequency. The autocorrelation function is therefore given by, $C(t)=d^{2} \int P\left(t_{0}\right) U\left(t<t_{0}\right)$ $\exp \left(-i \omega_{0} t\right) d t_{0}$, where $P\left(t_{0}\right)$ is the probability that a collision will occur after a time $t_{0}$, and $U\left(t<t_{0}\right)$ is a step function which equals 1 before the collision occurs (i.e., when $t<t_{0}$ ) and equals zero after the collision. Although the line shape is not always given in this form (cf. eq (1.2) of ref. [16] noting that $P\left(t_{0}\right)=\nu_{c}$ $\left.\exp \left(-\nu_{c} t_{0}\right)\right)$, the results are the same. In another classical theory Weisskopf, and later Lindholm, assumed that collisions can change the phase of the light train $[16,17]$. The line shape is again given by the Fourier transform of the light train autocorrelation function (see also eq (1.13a) of ref. [16] or eq (11.13) of ref. [17]) hence a phase shift decreases the phase correlation thereby broadening the Fourier spectrum (line shape). In these phase shift theories, $d(t)$ has the form $\exp \left[-i \omega_{0} t-i \eta(t)\right]$ where $\eta(t)$ represents the phase shifts produced by collisions. The autocorrelation function is $C(t)=d^{2} \exp \left(-i \omega_{0} t\right)\langle\exp [-i \eta(t)]\rangle$ where $\langle\ldots\rangle$ denotes an average over phase shifts (thermal average). The Weisskopf theory assumed that small phase shifts (less than unity) have no effect on $C(t)$, whereas large phase shifts (greater than unity) destroy the phase correlation of the light train and cause $C(t)$ to fall to zero. This effect is only apparent after one performs an average over a probability distribution of phase shifts. To show this, we consider a collision which occurs in the time interval $(t, t+\Delta t)$ and produces a phase shift $\eta_{0}$. If the duration of this collision $\tau$ is very short, $\tau<\Delta t$, then we have $C(t+\Delta t)$ $=C(t)\left\langle\exp \left(-i \eta_{0}\right)\right\rangle$ (the two factors are independent and are averaged separately as in the impact approximation; see sec. (11.3.2) of ref. [17]), where $\langle\exp$ $\left.\left(-i \eta_{0}\right)\right\rangle=\int \exp \left(-i \eta_{0}\right) P\left(\eta_{0}\right) d \eta_{0}$ and $P\left(\eta_{0}\right)$ denotes the probability of finding a phase shift of magnitude $\eta_{0}$. If $P\left(\eta_{0}\right)$ does not vary rapidly, then the rapid oscillation of $\exp \left(-i \eta_{0}\right)$ for $\eta_{0}>1$ will produce

$$
\int_{1}^{\infty} \exp \left(-i \eta_{0}\right) P\left(\eta_{0}\right) d \eta_{0} \ll 1
$$

(the integral from $-\infty$ to -1 is also much less than 1) whereas the integral from -1 to +1 is on the order of unity. Thus, for $\eta_{0}>1$, we have $C(t+\Delta t) \simeq 0$ and for $\eta_{0}<1, C(i+\Delta t) \simeq C(t)$. While the average over $\eta_{0}$ is given more accurately by later phase shift theories (e.g., Lindholm's theory), the qualitative results for the line shape stated by Weisskopf for $\eta_{0}>1$ are basically unchanged. It is in this sense that we will regard a large phase shift as an effective termination of the light train.

\section{b. Quantum Mechanical Theory}

In the quantum mechanical problem the radiators in a gas emit a series of spectral lines which are broadened due to the perturbation $V$. We are usually interested in just one of these broadened lines corresponding to some given unperturbed frequency $\omega_{0}$. The photons which contribute to this particular line are emitted in radiative transitions between some particular initial and final energy levels of the radiator (which may be perturbed). In this case, the light train is the wave function for a photon emitted in such a transition; the amplitude of the light train gives the probability of finding a photon emitted after some time $t$. Baranger has shown that the fünction $C(t)$ defined in eq (8) may be interpreted as the autocorrelation function for the light train amplitude (see p. 498 of ref. [4]). Thus the line shape is obtained from the Fourier transform of the light train autocorrelation function just as in the classical theories.

The effects of a collision may be roughly divided into two categories: adiabatic and nonadiabatic. We will discuss these separately although a single collision may have both effects. An adiabatic collision will simply shift the energy levels thereby producing a phase shift (which may be time dependent) in the time development operator $T(t)$. Such collisions will reduce the phase correlation between $\mathbf{d}(0)$ and $\mathbf{d}(t)=$ $T^{\dagger}(t) \mathbf{d} T(t)$ just as the collisions in the classical phase shift theories. A nonadiabatic collision will induce a radiationless transition out of the initial state. This type of transition usually "terminates" the probability that the radiator will emit a photon with a frequency near $\omega_{0}$. However, if there is another state whose energy is very close to that of the initial state, nonadiabatic collisions may simply produce transitions back and forth between these two states without completely destroying the probability of emission near $\omega_{0}$. The latter situation is usually referred to as the problem of overlapping lines (although it is sometimes called a "phase-memory" effect) [18]. In a classical oscillator having more than one mode of oscillation, a nonadiabatic collision simply changes the oscillation from one mode to another. The Lorentz interruption theory implicitly assumes that the mode whose frequency is $\omega_{0}$ is the only mode of interest and a (nonadiabatic) collision terminates this mode. However, if there were another mode with a frequency very close to $\omega_{0}$, nonadiabatic collisions could switch the oscillator back and forth between these two modes without destroying the phase coherence of the light train (overlapping line case). For example, an oscillator in the mode $\omega_{0}$ will have a phase $\omega_{0} t$ at some time $t$ (apart from an 
arbitrary initial phase). If this oscillator is switched to the mode $\omega_{1}$ at some time $t$ and, after a time $t_{0}$, is switched back to $\omega_{0}$, the resulting phase shift in the natural oscillation is $\eta \simeq\left[\omega_{0}\left(t+t_{0}\right)-\left(\omega_{0} t+\omega_{1} t_{0}\right)\right]=$ $\left(\omega_{0}-\omega_{1}\right) t_{0}$. Estimating $t_{0}$ by the mean free time $\left(1 / \nu_{c}\right)$ and noting that the collision frequency $\nu_{c}$ may be identified with the halfwidth $\Delta \omega_{1 / 2}$, (in a Lorentz picture), we have $\eta \simeq\left(\omega_{0}-\omega_{1}\right) / \Delta \omega_{1 / 2}$. If $\left(\omega_{0}-\omega_{1}\right)>\Delta \omega_{1 / 2}$ (isolated lines), we have $\eta>1$ and we may say that the light train is terminated by nonadiabatic collisions (as the Lorentz theory assumes). If $\left(\omega_{0}-\omega_{1}\right)<\Delta \omega_{1 / 2}$ (overlapping lines), we may say that the light train has retained a "memory of its initial phase." Thus we see that nonadiabatic collisions are analogous to those considered by the Lorentz interruption theory only in the case of isolated lines. In the case of overlapping lines, nonadiabatic collisions are not treated by any classical theories although the classical analog is obvious. We also note that a collision which produces both adiabatic and nonadiabatic effects cannot be conveniently treated by classical theories either.

To make our discussion of the quantum mechanical $C(t)$ more quantitative we will consider the case of a quantitative three-state radiator having a ground state with energy $E_{0}$ and two excited states with energies $E_{1}$ and $E_{2}$. We will also assume that the ground state is not perturbed by the interaction $V(t)$ and we use $V_{00}=V_{01}$ $=V_{02}=0$ (as well as $V_{i j}=V_{j i}$ ). A generalization to more complicated cases may be achieved by means of an obvious extension of the following arguments. The time development operator for the radiator (see eq (39)) will be written in an interaction representation defined by

$$
T_{a}(t)=\exp \left(-i t H_{a} / \hbar\right) T^{\prime}(t)
$$

where

$$
\begin{aligned}
T^{\prime}(t) & =\mathscr{O} \exp \left[-(i / \hbar) \int_{0}^{\tau} V^{\prime}\left(t^{\prime}\right) d t^{\prime}\right], \\
V^{\prime}(t) & =\exp \left(i t H_{a} / \hbar\right) V(t) \exp \left(-i t H_{a} / \hbar\right),
\end{aligned}
$$

and $\mathcal{O}$ is the time ordering operator [19]. Notice that $V_{0 j}=V_{j 0}=0$ implies that $T_{00}^{\prime}=1$ and $T_{0 j}^{\prime}=T_{j 0}^{\prime}=0$ for $j \neq 0$; this simplification is the reason for neglecting ground state interactions.

If we are interested only in the line whose unperturbed frequency is $\omega_{10}=\left(E_{1}-E_{0}\right) / \hbar$, we do not need all of the terms in the trace over states of the radiator (see eq (11)). We need only consider the terms

$$
\begin{aligned}
C(t)=\sum_{j} d_{j 0} d_{01}\left\langle T_{1 j}^{\prime}\right\rangle \rho_{j}^{(a)} \exp \left(-i \omega_{10} t\right) \\
+\sum_{j} d_{j 0} d_{02}\left\langle T_{2 j}^{\prime}\right\rangle \rho_{j}^{(a)} \exp \left(-i \omega_{20} t\right),
\end{aligned}
$$

where $\rho_{j}^{(a)} \propto \exp \left(-E_{j} / k T\right)$. The natural oscillation exp $\left(-i \omega_{10} t\right)$ in the first term may be regarded as an unperturbed light train which causes the Fourier transform of this term (eq (7)) to be large in the vicinity of $\omega_{10}$. Thus, when calculating the line $\omega_{10}$ we need only consider those terms in eq (11) whose natural oscillation is close to $\omega_{10}$. The second term in eq (51) describes radiation near $\omega_{20}$; this term will be important (when calculating the $\omega_{10}$ line) only if the lines $\omega_{10}$ and $\omega_{20}$ overlap (i.e., if their halfwidths are on the order of $\left.\left(\omega_{10}-\omega_{20}\right)\right)$. It will be shown later in this section that the phase of the off-diagonal terms such as $T_{12}^{\prime}$ varies according to the line spacing $\omega_{12}$; thus when the average time between collisions (which is on the order of $\left.1 / \Delta \omega_{1 / 2}\right)$ is small compared with $1 / \omega_{12}$, these off-diagonal terms will become important and phase memory effects (overlapping lines) will have to be considered. If $\omega_{10}$ and $\omega_{20}$ are isolated lines (i.e., their halfwidths are much smaller than $\omega_{12}$ ), the second term in eq (5l) (as well as the off-diagonal $T^{\prime}$ matrix elements) may be neglected. For some lines in the microwave region it may happen that the halfwidth of $\omega_{10}$ is on the order of $\omega_{10}$; in this case it would be necessary to add the "negative resonance" terms, exp $\left(-i \omega_{01} t\right)$, to eq (51).

If we factor the natural oscillation out of $C(t)$, we may write $C(t)=\exp \left(-i \omega_{10} t\right) \tilde{C}(t)$ where $\tilde{C}(t)$ is a decreasing function of $t$ [15]. The line shape (see eq (7)) is proportional to $\int \exp ($ it $\Delta \omega) \widetilde{C}(t) d t$ where $\Delta \omega=\left(\omega-\omega_{10}\right)$. Since exp $($ it $\Delta \omega)$ oscillates rapidly for $t>1 / \Delta \omega$, the intensity at the point $\Delta \omega$ is determined primarily by the part of $\tilde{C}(t)$ for which

$$
0 \leqslant t \leqslant 1 / \Delta \omega
$$

For this reason, $1 / \Delta \omega$ is frequently referred to as the time of interest in evaluating the intensity at the point $\Delta \omega$.

If the duration of a collision is much longer than the time of interest, the perturber may be regarded as stationary during this time. This quasi-static approximation is frequently employed in the description of ions in a plasma $[4,6]$. Such static perturbations simply add a constant to the oscillation frequencies $\omega_{10}, \omega_{20}$, etc. The influence of dynamic perturbations, for which the collision time is less than or the order of the time of interest, is obtained by calculating $\left\langle T_{1 j}^{\prime}\right\rangle$ and $\left\langle T_{2 j}^{\prime}\right\rangle$.

We are interested in the classical correspondence for the quantum mechanical $C(t)$ primarily as an aid in establishing a criterion for the so-called strong collisions (this will be done in sec. 7.3). When a single collision is capable of reducing $C(t)$ to zero, it is referred to as a strong collision. To study this effect we need only consider the influence of a single collision on $C(t)$. We will consider some particular type of collision (e.g., given values of impact parameter, velocity, etc.) which is described by the interaction potential $V(t)$. We will assume that this is the first collision to occur after some arbitrarily chosen starting time $t=0$ (if a collision does not reduce $C(t)$ to zero, it is "weak," and to find a strong collision criterion we may neglect the effects of all previous "weak" collisions). The collision will begin at some time $t_{0} \geqslant 0$, and we define a collision time $\tau$ such that $V(t)=0$ unless $t_{0} \leqslant t \leqslant t_{0}+\tau$. From eq (50) we see 
that $T^{\prime}(t)=1$ when $t<t_{0}$; when $t>\left(t_{0}+\tau\right)$, we change the variable of integration to $t^{\prime \prime}=\left(t^{\prime}-t_{0}\right)$ and obtain

$$
T^{\prime}(t)=\exp \left(i t_{0} H_{a} / \hbar\right) S \exp \left(-i t_{0} H_{a} / \hbar\right),
$$

where $S$ is the $S$-matrix for the collision,

$$
\begin{aligned}
S=1-(i / \hbar) & \int_{0}^{t} \tilde{V}(t) d t \\
& -(1 / \hbar)^{2} \int_{0}^{\tau} \int_{0}^{t} \tilde{V}(t) \tilde{V}\left(t^{\prime}\right) d t^{\prime} d t+\cdots \cdot
\end{aligned}
$$

and

$$
\tilde{V}(t)=\exp \left(i t H_{a} / \hbar\right) V\left(t+t_{0}\right) \exp \left(-i t H_{a} / \hbar\right) .
$$

The contribution to $C(t)$ from this collision will be called $C(V(t), t)$; this function will have the same form as $C(t)$ in eq (51) except that there will be no average $\langle\ldots\rangle$ over collisions. To obtain $C(t)$ we would simply average $C(V(t), t)$ over the collision parameters for the first collision (and all successive collisions if the first collision is not strong).

\section{c. Isolated Lines}

If the lines $\omega_{10}$ and $\omega_{20}$ are isolated, $C(V(t), t)$ is given by $\Sigma_{j} d_{j o} d_{01} T_{1 i}^{\prime} \rho_{i}^{(a)} \exp \left(-i \omega_{10} t\right)$. In this series $T_{10}^{\prime}=0$ (no ground state interaction) and $T_{12}^{\prime}$ is unimportant (for isolated lines only). As mentioned earlier, to show that $T_{12}^{\prime}$ is unimportant it is necessary to average over $t_{0}$. This has been done for the line wings in eq (19) of ref. [20] and the second term in this equation, which contains the off-diagonal elements of the time development operator, is negligible in the case of isolated lines (see also the following section on overlapping lines). Using this fact we have

$$
C(V(t), t) \propto d_{10}^{2} \rho_{1} T_{11}^{\prime}(t) \exp \left(-i \omega_{10} t\right) .
$$

Since the complex function $T_{11}^{\prime}(t)$ can always be written in the classical form $\exp [-i \eta(t)-\epsilon(t)]$, the classical correspondence for $C(V(t), t)$ becomes obvious. Collisions for which $\epsilon=0$ are equivalent to the collisions in the classical phase shift theories and those for which $\epsilon \gtrsim 1$ are equivalent to the nonadiabatic collisions in the Lorentz theory. To illustrate the functional form of $\eta$ and $\epsilon$, we will evaluate $T_{11}^{\prime}(t)$, for $t>\left(t_{0}+\tau\right)$, to lowest order in $V(t)$. We will use $V_{11}=V_{22}=0$ since $V$ has no diagonal matrix elements in most problems (i.e., $V$ usually has nonvanishing matrix elements only between states with different angular momenta). Using eqs (53), (54), and (55) we obtain

$$
\begin{array}{r}
T_{11}^{\prime}(t)=1-\hbar^{-2} \cdot \int_{0}^{\tau} \int_{0}^{t} V_{12}\left(t+t_{0}\right) V_{21}\left(t^{\prime}+t_{0}\right) \\
\exp \left\{i \Delta E_{21}\left(t^{\prime}-t\right) / \hbar\right\} d t^{\prime} d t
\end{array}
$$

$$
\simeq 1-i\left(\bar{V}_{12}^{2} / \hbar \Delta E_{21}\right) \int_{0}^{\tau}\left[\exp \left\{i t \Delta E_{21} / \hbar\right\}-1\right] d t,
$$

where $\Delta E_{21}=\left(E_{2}-E_{1}\right)$ and $\bar{V}_{21}$ denotes the average value of $V_{21}(t)$ during the collision. For fast collisions $\left(\Delta E_{21} \tau / \hbar\right) \ll 1$, we have

$$
T_{11}^{\prime}(t) \simeq 1-\frac{1}{2}\left(\bar{V}_{21} \tau / \hbar\right)^{2} .
$$

For slower collisions $\left(\Delta E_{21} \tau / \hbar\right) \gg 1$, we replace $\tau$ by $\infty$ when integrating the exponential in eq (57). We then replace $\Delta E_{21}$ by $\left(\Delta E_{21}+i \delta\right)$, perform the integral, and then take the limit $\delta \rightarrow 0$. The justification for this procedure is the "radiation damping" which produces the natural line width (see sec. 13.A of ref. [3]). In this manner we obtain

$$
T_{11}^{\prime}(t) \simeq 1+i\left(\bar{V}_{21}^{2} \tau / \hbar \Delta E_{21}\right) .
$$

These results may be summarized by

$$
C(V(t), t) \propto d_{10}^{2} \rho_{1} \exp \left(-i \omega_{10} t+i \eta-\epsilon\right),
$$

where

$$
\eta=\left\{\begin{array}{cc}
0 & \left(\Delta E_{21} \tau / \hbar\right) \ll 1 \\
\left(\bar{V}_{21}^{2} \tau / \hbar \Delta E_{21}\right) & \left(\Delta E_{21} \tau / \hbar\right) \gg 1
\end{array}\right.
$$

and

$$
\epsilon=\left\{\begin{array}{cl}
\frac{1}{2}\left(\bar{V}_{21} \tau / \hbar\right)^{2} & \left(\Delta E_{21} \tau / \hbar\right) \ll 1 \\
0 & \left(\Delta E_{21} \tau / \hbar\right) \gg 1 .
\end{array}\right.
$$

Notice that if the perturbation is not strong enough to mix adjacent levels, $\bar{V}_{21} \ll \Delta E_{21}$, then $\epsilon \ll 1$ and we have only a phase shift. That is, all collisions are adiabatic if $\bar{V}_{21} \ll \Delta E_{21}$. If adjacent levels are mixed, we will have phase shifts produced by slow (adiabatic) collisions and inelastic transitions produced by fast (nonadiabatic) collisions.

For a series of collisions which do not overlap in time (or for which this overlap is negligible) the classical form is preserved and the net result is a phase shift $\eta$ and a damping $\epsilon$ which are obtained by adding up the phase shifts and damping constants from each of the collisions. When two collisions overlap, quantum mechanical mixing effects occur and the classical form is destroyed.

\section{d. Overlapping lines}

If the lines $\omega_{10}$ and $\omega_{20}$ are not isolated we must consider all terms in eq (51):

$$
\begin{aligned}
& C(V(t), t) \propto\left[\rho_{1} d_{10}^{2} T_{11}^{\prime}+\rho_{2} d_{20} d_{01} T_{12}^{\prime}\right] \exp \left(-i \omega_{10} t\right) \\
& \quad+\left[\rho_{2} d_{20}^{2} T_{22}^{\prime}+\rho_{1} d_{10} d_{02} T_{21}^{\prime}\right] \exp \left(-i \omega_{20} t\right) .
\end{aligned}
$$

The $\rho_{1} T_{11}^{\prime}$ term gives the probability that a radiator initially in the state $E_{1}$ will remain in that state after a time $t$; the $\rho_{2} T_{12}^{\prime}$ term gives the probability that a radia- 
tor initially in the state $E_{2}$ will make a radiationless transition to $E_{1}$ during the time $t$; together these two terms describe the probability, at the time $t$, that a radiator will emit a photon with a frequency near $\omega_{10}$. The remaining terms in eq (63) describe the probability of emission near $\omega_{20}$ (we must consider these terms when the lines $\omega_{10}$ and $\omega_{20}$ overlap).

When $t<t_{0}$, the radiator is unperturbed hence $T_{11}^{\prime}=T_{22}^{\prime}=1$ and $T_{12}^{\prime}=T_{21}^{\prime}=0$; in this case, $C(V(t), t)$ $\propto\left[\rho_{1} d_{10}^{2} \exp \left(-i \omega_{10} t\right)+\rho_{2} d_{20}^{2} \exp \left(-i \omega_{20} t\right)\right]$. When the collision occurs, we get a probability for switching between the modes $\omega_{10}$ and $\omega_{20}$ as discussed earlier; that is, $T_{12}^{\prime}$ and $T_{21}^{\prime}$ increase in amplitude while $T_{11}^{\prime}$ and $T_{22}^{\prime}$ decrease. To show this effect, we will calculate the $T^{\prime}$ matrix elements for $t>\left(t_{0}+\tau\right)$.

The condition for overlapping lines is $\left(\Delta E_{21} / \hbar\right)$ $\lesssim \Delta \omega_{1 / 2} ;$ since we may identify $\Delta \omega_{1 / 2}$ with $\nu_{c}$ where $\left(1 / \nu_{c}\right)$ is the mean free time between collisions and since $\tau$ is usually much less than $1 / \nu_{c}$ (at least for strong collisions), we must have $\left(\Delta E_{21} \tau / \hbar\right) \ll 1$. Noting the results of eqs (57) and (58), we see that when $\left(\Delta E_{21} \tau / \hbar\right) \ll 1$, the $S$ matrix elements may easily be evaluated to all orders with the following results:

$$
\begin{aligned}
& S_{11}=S_{22}=\cos \left(\bar{V}_{21} \tau / \hbar\right) \\
& S_{12}=S_{21}=-i \sin \left(\bar{V}_{21} \tau / \hbar\right) .
\end{aligned}
$$

Using eqs (55), (63), and (64) we may give an explicit expression for $C(V(t), t)$ in terms of $\left(\bar{V}_{21} \tau / \hbar\right)$. To simplify the following discussion we will use $C(V(t), t)$ $=C_{1}(V(t), t)+C_{2}(V(t), t)$, where

$$
\begin{aligned}
& C_{1}(V(t), t) \propto \rho_{1}\left[d_{10}^{2} \exp \left(-i \omega_{10} t\right) \cos \left(\bar{V}_{12} \tau / \hbar\right)\right. \\
& \left.\quad-i d_{20}^{2}\left(d_{10} / d_{20}\right) \exp \left(-i \omega_{20} t+i \omega_{21} t_{0}\right) \sin \left(\bar{V}_{21} \tau / \hbar\right)\right],
\end{aligned}
$$

and $C_{2}(V(t), t)$ contains the two $\rho_{2}$ terms in eq (63). $\left(C_{2}\right.$ may be obtained by interchanging the indices 2 and 1 in $C_{1}$.) We will discuss only $C_{1}$ since the interpretation of $C_{2}$ is obviously identical.

$C_{1}(V(t), t)$ describes the behavior of a radiator which was initially in the state $E_{1}$. Before the collision

$$
C_{1}(V(t), t) \propto \rho_{1} d_{10}^{2} \exp \left(-i \omega_{10} t\right)
$$

and after the collision, $t>t_{0}+\tau, C_{1}$ is given by eq (65). $\cos ^{2}\left(\bar{V}_{21} \tau / \hbar\right)$ gives the probability that the radiator will be in the state $E_{1}$ after the collision, and $\sin ^{2}$ $\left(\bar{V}_{21} \tau / \hbar\right)$ gives the probability that the collision will induce a radiationless transition to the state $E_{2}$. If $\left(\bar{V}_{12} \tau / \hbar\right)=(\pi / 2)$, the collision will definitely cause a transition to $E_{2}$ and we would have

$$
C_{1}(V(t), t) \propto \rho_{1} d_{20}^{2}\left(d_{10} / d_{20}\right) \exp \left(-i \omega_{20} t+i \omega_{21} t_{0}\right)
$$

after the collision. Equation (67) describes a light train for the line $\omega_{20}$ with a phase shift $\omega_{21} t_{0}$ just as in the classical oscillator discussed earlier (the factor $d_{10} / d_{20}$ represents the arbitrary initial phase difference between the two modes as well as an amplitude difference if one should exist). This result is identical with the result for a two mode classical oscillator because, when $\left(\bar{V}_{12} \tau / \hbar\right)$ equals some odd multiple of $(\pi / 2)$, there is definitely a change of mode; for other values of $\left(\bar{V}_{12} \tau / \hbar\right)$, the quantum mechanical expression, eq (65), shows only a probability for this mode switching effect. Notice that the mode switching or phase-memory effects are described by the offdiagonal $T^{\prime}$ matrix elements. These off-diagonal elements were not needed in the description of isolated lines because the average phase shift $\omega_{21} t_{0}$ is greater than unity (recall that the average value of $t_{0}$ is the mean free time $\left(1 / \nu_{c}\right)$ which is estimated by $\left.1 / \Delta \omega_{1 / 2}\right)$ and these elements vanish after the $t_{0}$ average.

In the case of overlapping lines, $C(V(t), \mathrm{t})$ does not have the simple classical form which was obtained for isolated lines. In this case we would average $C(V(t), t)$ over collision parameters by defining a quantity $\epsilon=\left(\bar{V}_{12} \tau / \hbar\right)$ and a function $P(\epsilon)$ which gives the probability that the collision will produce a $\left(\bar{V}_{12} \tau / \hbar\right)$ whose magnitude is $\epsilon$. The average over $C(V(t), t)$ contains terms like

$$
\int P(\epsilon) \cos \epsilon d \epsilon \text { and } \int P(\epsilon) \sin \epsilon d \epsilon \text {. }
$$

If $P(\epsilon)$ does not vary rapidly then we may say that collisions for which $\epsilon>1$ effectively terminate the wave train (this argument is analogous to the treatment of large phase shifts in the classical theories).

\subsection{Strong Collisions}

In discussing the approximations relevant to the density matrix and the wave functions, our general procedure will be to show that each approximation breaks down only during a "strong collision." It is thus convenient at this point to clarify our meaning of strong collisions and to discuss briefly the dynamics of certain types of collisions.

In the previous section it was shown that each collision produces a phase shift and changes the amplitude of the light train. In this manner, a series of collisions will eventually destroy the correlation between $d(t)$ and $d(0)$ and reduce $C(t)$ to zero. It may happen that a single collision is capable of reducing $C(t)$ to zero; such an event is defined to be a strong collision. From the results of the previous section we see that a strong collision is one which produces a large phase shift, $\eta>1$, or a large decrease in amplitude, $\epsilon>1$. Such collisions terminate the light train and therefore satisfy the hypotheses of the Lorentz interruption theory when $\epsilon>1$, or Weisskopfs phase shift theory when $\eta>1$. The general program of classical path theories is to describe all but the strong collisions assuming that these will be treated by some other method such as a LorentzWeisskopf approximation [21].

From the results of the previous section, we see that a strong collision will occur when 


$$
(V \tau / \hbar) \gtrsim 1
$$

unless the level spacing is greater than $V$ (cf eq. (61) using $\Delta E_{21}>\bar{V}_{21}$ ). If $\Delta E_{21}>\bar{V}_{21}$ we must replace the $V$ in eq (68) by $V_{\text {eff }}=\bar{V}_{21}^{2} / \Delta E_{21}$; in this case, $V_{\text {eff }}$ is the actual perturbation of the radiator since we have used $V_{11}=0$. It is in this sense that we use eq $(68)$ as a working definition of strong collisions. It should be noted that our definition of a strong collision as one which terminates the light train is basically equivalent to the strong collisions in the impact theory [21] which are said to occur when the terms in the $S$-matrix expansion (see eq (54)) are on the order of unity or larger.

Most classical potential functions are inversely proportional to some power of the perturber position $\mathbf{r}(t) \simeq \boldsymbol{\rho}_{0}+\int_{0}^{t} \mathbf{v}\left(t^{\prime}\right) d t^{\prime}$, where $\boldsymbol{\rho}_{0}$ is the radiator-perturber separation at the time of closest approach and $\mathbf{v}(t)$ is the instantaneous perturber velocity. The maximum value of the potential where $|\mathbf{r}(t)|$ is smallest, namely when $\mathbf{r}(0)=\boldsymbol{\rho}_{0}$. The half maximum occurs when $|\mathbf{r}(\tau)|=\left|\boldsymbol{\rho}_{0}+\int_{0}^{\tau} \mathbf{v}\left(t^{\prime}\right) d t^{\prime}\right| \simeq 2 \rho_{0}$ (which may be taken as a definition of the collision time $\tau$ ). If $\mathbf{v}(t)$ changes very little during a collision we may use $\mathbf{v}(\tau) \simeq \mathbf{v}(0)=\mathbf{v}_{0} \quad$ or $\quad|r(\tau)| \simeq\left|\boldsymbol{\rho}_{0}+\tau \mathbf{v}_{0}\right| \simeq 2 \rho_{0}$. Since $\boldsymbol{\rho}_{0} \cdot \mathbf{v}_{0}=0$, the collision time is on the order of

$$
\tau=\rho_{0} / v_{0}
$$

where $v_{0}$ is the velocity at the time of closest approach. It can be shown by a rather lengthy analysis of hyperbolic trajectories, that this estimate is valid even when the radiator is an ion which accelerates a charged perturber.

If the radiator is electrically neutral, we may estimate $v_{0}$ by the thermal velocity $v_{\mathrm{av}}=\sqrt{3 k T / m}$. If both radiator and perturber are charged particles, we may estimate $v_{0}$ by

$$
v_{0}^{2}=v_{\mathrm{av}}^{2}-2 Z_{r} Z_{p} e^{2} / m \rho_{0}
$$

where $Z_{r}$ and $Z_{p}$ denote the charge of the radiator and perturber respectively.

\section{Validity Criteria}

\subsection{General}

An expression for the "thermal average" has been derived, eq (47), using a classical path treatment. This expression, or one like it, is the starting point for most of the modern semiclassical line broadening theories and it may be regarded as the end result of the classical path approximation. It is therefore worthwhile to examine the validity criteria for this expression.

It has been shown that the classical path approximation, as it is used in line broadening theories, actually embodies several types of approximations. As we have stated them, they may be roughly divided into three classes; (1) the representation of the gas by a collection of noninteracting cells, (2) simplifications of the density matrix, and (3) a classical treatment of the perturber wave functions.

In order to justify the mathematical model which is used by most line broadening theories, we have assumed that the average spacing between radiators is large enough that all direct and indirect interactions between radiators are negligible. (By indirect interactions we mean that one radiator may influence a perturber which is interacting with another radiator.) It is probably possible to justify the mathematical model with more relaxed assumptions, however, since our assumptions are satisfied for virtually all problems of interest, this may not be necessary. For resonance broadening in neutral gases, this approximation may have to be reconsidered.

It should be noted that the radiator Hamiltonian $H_{a}$ should describe translational motion of the radiators as well as their internal structure (i.e., bound states). In practice, however, one usually neglects this translational motion and calculates only the discrete spectrum. This procedure neglects Doppler shifts which also broaden a given line. The Doppler broadening may be reinstated, in an approximate manner, by means of convolution integrals [6]. This folding procedure should be valid if the radiators trajectory, during the time of interest, is not appreciably altered by collisions with other particles. For most plasma line broadening problems this criterion is well satisfied however, for some foreign gas broadening problems one may have to treat Doppler effects more carefully.

\subsection{Density Matrix}

The approximations relevant to the density matrix are for the purpose of achieving a product form, eq (8), which permits us to factor the trace operations, as in eq (11), and thereby perform the average over perturber states without affecting the sum over states of the radiator. In this weak coupling approximation, the subsystems are statistically independent and this is what is meant by the neglect of back reaction as it applies to the statistics (density matrix). This approximation has been made by replacing the term exp $\{-V \mid k T\}$ in the Boltzmann factor by unity; that is, we have assumed that all $V$ matrix elements (or at least those which pertain to the spectral line of interest) will satisfy $V \ll k T$. It should be noted that in obtaining this inequality we have tacitly assumed that the radiators and perturbers are in thermal equilibrium at a temperature $T$. For many line broadening experiments, however, the radiators and perturbers have different temperatures. Nonetheless, if the perturbers are in thermal equilibrium at a temperature $T_{p}$, the weak coupling approximation will still be valid for $V \ll k T_{p}$ and the perturber statistics will be described by a Boltzmann factor as in eq (47). In such a case, the statistical weights for the atomic states (matrix elements of $\rho^{(a)}$ ) would be determined from the rate equations which describe the population and depopulation of atomic levels. We will henceforth take 
$V \ll k T$, where $T$ is the perturber temperature, as the validity criterion for the weak coupling approximation. It is obvious that this inequality will be violated for some interactions, however, it will be shown that the errors made by this approximation are important only in the far wings of a line where $\hbar \Delta \omega \gtrsim k T$.

If the perturbation $V \gtrsim k T$ is produced by a static ion (one which does not move appreciably during the time of interest, see eq (52)) in a plasma, the Stark splitting is on the order of $k T$, and it is immediately obvious that this will influence only the far wings, $\Delta \omega>k T / \hbar$, of a line.

When the perturbation $V>k T$ is produced during a collision with a dynamic perturber (we will consider electrons as representatives of dynamic perturbers), the radiator-perturber separation must be on the order of $\chi_{\mathrm{av}}=\hbar / \sqrt{3 m k} T$ (the thermal wavelength, or average de Broglie wavelength, for the perturber) or smaller [22]. Using $V=k T, \rho_{0}=\chi_{\text {av }}$ and $v_{0}=v_{\text {av }}$ in eq (68), we see that, for neutral radiators, $V \gtrsim k T$ can occur only during a strong collision (for most neutral radiators the energy spacing between adjacent states is less than $k T$ and it is not necessary to consider $V_{\text {eff }}$ as discussed in sec. 7.3). If a charged perturber collides with an oppositely charged radiating ion, it will be accelerated by the Coulomb attraction $V_{0}$, hence $v_{0}>v_{\text {av }}$. Using $V=k T, \rho_{0}=\chi_{\text {av }}$, and $v_{0}=v_{\text {av }}\left(v_{0} / v_{\text {av }}\right)$ in eq $(68)$, we find that $(V \tau / \hbar)$ is on the order of $\left(v_{\text {av }} / v_{0}\right)$, which is less than 1. Thus, in this case, $V \gtrsim k T$ need not correspond to a strong collision. In a collision involving a radiating ion and a charged perturber of like sign, the perturber will be decelerated, and it is unlikely that it will come close enough to the radiator to produce $V \gtrsim k T$. To show this, we note that the smallest value of $\rho_{0}$ given by eq $(70)$ is $\left(2 e^{2} / m \rho_{0}\right)=v_{\text {av }}^{2}$ or $\rho_{0}=\chi_{\text {av }}\left(2 e^{2} / \hbar\right)$ $\sqrt{m / 3 k T}$; in order that $\rho_{0}$ be on the order of $\chi_{\mathrm{av}}$, it is necessary that $T$ be larger than $10^{8} \mathrm{deg} \mathrm{K}$.

Regarding $C(t)$ as a weighted sum of $\mathbf{d} \cdot \mathbf{d}(t)$ terms as discussed in section 7.1, it is clear that the approximate density matrix $\rho^{(a)} \rho^{(p)}$ will incorrectly weight only those $\mathbf{d} \cdot \mathbf{d}(t)$ terms which correspond to a radiator initially undergoing a $V \gtrsim k T$ collision (i.e., the density matrix weights each term according to the state at $t=0$ ). This does not preclude the possibility that $V \gtrsim k T$ collisions may occur at later times, $t>0$, in the remaining, correctly weighted, $\mathbf{d} \cdot \mathbf{d}(t)$ terms. Such events will indeed occur, however, they are determined by the dynamics of the system and this has no connection with the statistical weighting (density matrix) of the corresponding initial states. If we define a collision frequency $\nu_{k T}$ for the $V \gtrsim k T$ collisions then the probability that such a collision will occur within a time $\tau$ of $t=0$ is $\nu_{k T} \tau$. The number of incorrectly weighted $\mathbf{d} \cdot \mathbf{d}(t)$ terms will therefore be $\nu_{k T} \tau N_{T}$, where $N_{T}$ is the total number of terms. The number of correctly weighted terms will be $\left(1-\nu_{k T} \tau\right) N_{T}$, and the error resulting from the use of the weak coupling density matrix will be on the order of $\nu_{k T} \tau /\left(1-\nu_{k T} \tau\right)$. Since the duration, $\tau$, of a $V \gtrsim k T$ collision will be much smaller than the time between such collisions, we may replace $\left(1-\nu_{k} \tau\right)$ by 1 and the error estimate becomes $\nu_{k T} \tau$. The error will be largest when all collisions produce $V \gtrsim k T$; in this case we may estimate the collision frequency by $\Delta \omega_{1 / 2}$, the halfwidth of the observed line. The largest value of $\tau=\rho_{0} / v_{0}$ for a $V \gtrsim k T$ collision occurs for $\rho_{0}=\chi_{\mathrm{av}}$ and $v_{0}=v_{\mathrm{av}}$ (decelerated perturbers, $v_{0}<v_{\text {av }}$, are not likely to produce $V \gtrsim k T$ ). The largest value of $\tau$ is $\hbar / k T$ hence the largest error is on the order of $\hbar \Delta \omega_{1 / 2} / k T$. Since $\hbar \Delta \omega_{1 / 2}$ is much less than $k T$ for virtually all lines of interest, this error is negligible. A more detailed analysis of the incorrectly weighted terms shows that this error estimate breaks down when $\Delta \omega \gg \Delta \omega_{1 / 2}$. In this case the time of interest is less than the average time between collisions (recall that $\Delta \omega_{1 / 2} \cong \nu_{k T}$ for the worst case), and some of the correctly weighted d.d $(t)$ terms will not suffer a collision during the time of interest. These "unperturbed" terms will have little influence on the line shape in the region $\Delta \omega>\Delta \omega_{1 / 2}$ (their dominant influence is in the line center when $\left.\Delta \omega<\Delta \omega_{1 / 2}\right)$. We must therefore compare the number of incorrectly weighted terms with $\left(\nu_{k T} / \Delta \omega\right)\left(1-\nu_{k T} \tau\right) N_{T}$ (the number of correctly weighted terms which have undergone a collision during the time of interest, $1 / \Delta \omega$ ). In this case the error estimate becomes $\hbar \Delta \omega / k T$ which is small if $\Delta \omega \ll k T / \hbar$. It must be emphasized that these error estimates are only an upper bound for the true error which, in most cases, is much less than $\left(\hbar \Delta \omega_{1 / 2} / k T\right)$ or $(\hbar \Delta \omega / k T)$.

For neutral radiators $V \gtrsim k T$ occurs only during a strong collision. Since a strong collision completely disrupts the light train, the weighted sum of all d $\cdot \mathbf{d}(t)$ terms which correspond to a strong collision at $t=0$ will have fallen to zero by the time these collisions are completed. That is, the halfwidth for the sum of all incorrectly weighted d $\cdot \mathbf{d}(t)$ terms will be on the order of $\tau=\hbar / k T$ or smaller. The corresponding Fourier transform will thus be a constant for $\hbar \Delta \omega<k T$. Since the incorrectly weighted terms add only a constant to $I(\omega)$ for $\hbar \Delta \omega<k T$, the approximate density matrix will not affect the shape of $I(\omega)$ for neutral radiators. Since most $V \gtrsim k T$ collisions are strong collisions even when the radiator is an ion, the above argument may be loosely applied to ion lines as well. We may therefore give the condition for validity of the weak coupling density matrix as

$$
\hbar \Delta \omega \ll k T \text {. }
$$

This condition was also obtained by Baranger [5,23] within the framework of a quantum mechanical impact theory. We note, in passing, that the region of validity for the impact approximation is given $\tau_{0} \Delta \omega \ll 1$ where $\tau_{0}$ is some representative collision time (see sec. 5.1 of ref. [4]). Since $\tau_{0} \gtrsim \hbar / k T$, we may state that when the impact approximation is valid the weak coupling density matrix may be used; the converse of this statement is not necessarily true.

\subsection{Wave Functions}

The dynamics of the system are needed only to calculate $\mathbf{d}(t)$ from $\mathbf{d}(0)$ for each given initial state; this requires a knowledge of $T(t)$ but not $\Psi(\mathbf{R}, \mathbf{x}, t)$. 
That is, since we are working with a trace, eq (8), it is not necessary to know the wave functions $\Psi(\mathbf{R}, \mathbf{x}, t)$ for the system (although it is necessary to define a set of basis functions). Semiclassical approximations have been made in the wave functions simply to permit the use of a classical potential function, $V^{(i)}\left(\mathbf{R}, \mathbf{x}^{(i)}, \mathbf{v}^{(i)}, t\right)$, in the time development operator. These semiclassical approximations, which are the hallmark of the classical path approximation, permit a simple physical interpretation of the dynamics of the system. More powerful quantum mechanical treatments are available $[3,4]$; however, in many cases, the results of these theories differ only slightly from the classical path treatment and the physical insight afforded by the latter is frequently advantageous.

The wave packet assumption was used to obtain the relation

$$
V(\mathbf{R}, \mathbf{x}) \varphi^{(i)}(\mathbf{x}, t)=V^{(i)}\left(\mathbf{R}, \mathbf{x}^{(i)}, \mathbf{v}^{(i)}, t\right) \varphi^{(i)}(\mathbf{x}, t),
$$

where $V^{(i)}$ is a classical function of the classical position and velocity coordinates $\mathbf{x}^{(i)}$ and $\mathbf{v}^{(i)} \cdot V(\mathbf{R}, \mathbf{x})$ can be expressed as a sum of interactions $V\left(\mathbf{R}, \mathbf{x}_{j}\right)$ between the radiator and each perturber (recall that $\left.\mathbf{x}=\left(\mathbf{x}_{1}, \mathbf{x}_{2}, \ldots \mathbf{x}_{N}\right)\right)$, thus we need only require that

$$
V\left(\mathbf{R}, \mathbf{x}_{j}\right) \varphi^{(i)}\left(\mathbf{x}_{j}, t\right)=V^{(i)}\left(\mathbf{R}, \mathbf{x}_{j}^{(i)}, \mathbf{v}_{j}^{(i)}, t\right) \varphi^{(i)}\left(\mathbf{x}_{j}, t\right)
$$

when $V\left(\mathbf{R}, \mathbf{x}_{j}\right) \neq 0$ in order to obtain eq (72). Messiah [24] has shown that eq (73) is obtained if the perturber is represented by a classical wave packet $[25,26]$ and if the potential field seen by the perturber does not vary appreciably (as compared with the perturbers kinetic energy) over the width of the wave packet.

The conditions for validity of eq (73) are discussed in detail by Messiah and we shall only outline this discussion as it applies to our problem. We must show; (1) that it is possible to construct a wave packet which does not overlap with the wave functions for the other particles, (2) that this wave packet does not spread appreciably during a collision (i.e., when $V\left(\mathbf{R}, \mathbf{x}_{j}\right)$ is nonzero), and (3) that the potential seen by the perturber does not vary appreciably over the width of a wave packet. The potential seen by the perturbers may be obtained by iterating the Schrödinger equations for $\chi^{(i)}$ and $\varphi^{(i)}$ to the next higher order as discussed in section 5 . This potential will be the matrix elements of $V(\mathbf{R}, \mathbf{x})$ between atomic states or, in some cases, it will be $V_{\text {eff }}$ as discussed in section 7.3, (or some other type of optical potential) [27]. The momentum of a perturber moving in the

potential $V(\mathbf{R}, \mathbf{x})$ is given by $p=\sqrt{2 m(E-V)}$, where $E$ denotes the perturber energy. From this expression we see that $\Delta p=m \Delta V / p$, where $\Delta p$ and $\Delta V$ denote the variation in $p$ and $V$ over the width of a wave packet. The condition that $\Delta V \ll p^{2} / 2 m$ is thus equivalent to

$$
\Delta p \ll p
$$

which is the usual condition for validity of WKB calculations (see eq (7.13) of ref. [28] noting that $\lambda(d p / d x)$ may be replaced by $\Delta p$ ). We will assume for the moment that this inequality can be satisfied, and it will be shown that the other validity conditions are easily met.

We wish to represent those perturbers which are not interacting with the radiator by Gaussian wavepackets of width $\Delta x_{g}$ and momentum spread $\Delta p_{g} \ll p_{\text {av }}$ at some instant of time. For this wave packet construction we must require that $\Delta x_{g}$ be much less than the average particle spacing $n^{-1 / 3}$ ( $n$ is the perturber density) so that the wave packets do not overlap. Using $\Delta x_{g} \Delta p_{g} \simeq \hbar$ (for a Gaussian wavepacket), we see that the condition $\Delta p_{g} \ll p_{\text {av }}$ also requires $\Delta x_{g} \gg \hbar / p_{\text {av }}$ $=\chi_{\mathrm{av}}$. We must therefore be able to satisfy.

$$
\chi_{\mathrm{av}} \ll \Delta x_{g} \ll n^{-1 / 3} \text {. }
$$

For virtually all problems of interest we have $\chi_{\text {av }} \ll$ $n^{-1 / 3}$, hence it will be possible to construct Gaussian wave packets for the overwhelming majority of perturbers at some instant of time.

Since static perturbers (e.g., ions in a plasma) are regarded as stationary during the time of interest, it is only necessary that they be represented by wave packets at some instant of time. As discussed above, this will be possible for the overwhelming majority of perturbers if $\hbar_{a v} \ll n^{-1 / 3}$. The treatment of static perturbers will be discussed further in II and for the remainder of this section we will consider only dynamic perturbers.

During a collision a wave packet will generally increase both its width, $\Delta x$, and its momentum spread, $\Delta p$. If $\Delta p \ll p$ (or $\Delta V<p^{2} / 2 m$, which will be verified shortly), the increase in momentum spread will be negligible, and for a collision of duration $\tau=\rho_{0} / v_{0}$, the wave packet will increase its width to a maxımum of $\Delta x_{\tau}=(\Delta x+\tau \Delta p / m)$. To prevent overlap with the radiators wave function during the collision $\Delta x_{\tau}$ should satisfy $\left(\Delta x_{\tau}+R_{0}\right)<\rho_{0}$, where $R_{0}$ denotes the effective "extent" of the perturber wave function. Using $\tau=\rho_{0} / v_{0}$ and $\Delta p \ll p$, we see that $(\tau \Delta p / m) \ll \rho_{0}$ hence we need onlv require $\left(\Delta x+R_{0}\right)<\rho_{0}$.

For a collision involving a neutral radiator the perturber momentum may be taken to be $p_{\text {av }}$, hence we may use $\Delta x \simeq \Delta x_{g}$ and $\Delta p \simeq \Delta p_{g}$ (which will then satisfy $\Delta p \ll p)$. Since $\Delta x$ may be within an order of magnitude of $\chi_{\mathrm{av}}$ (and still satisfy $\Delta x \gg \chi_{\mathrm{av}}$ ), we may obtain an order of magnitude estimate of the overlap condition, $\left(\Delta x+R_{0}\right)<\rho_{0}$, by considering $\left(\chi_{\mathrm{av}}+R_{0}\right)<\rho_{0}$. If $R_{0} \lesssim \mathcal{X}_{\mathrm{av}}$, the wave functions will overlap only when $\rho_{0} \lesssim \bar{X}_{\mathrm{av}}$, and for neutral radiators, this produces a strong collision (see sec. 8.2). If $R_{0} \gg \chi_{\mathrm{av}}$, a more detailed analysis of the perturbation potential is required; as an example we will consider the Stark broadening interaction, $V \simeq e^{2} R_{0} / \rho_{0}^{2}$, for which $(V \tau / \hbar) \simeq\left(R_{0} / \rho_{0}\right)\left(e^{2} / \hbar v_{\text {av }}\right)$. Since $\left(e^{2} / \hbar v_{\text {av }}\right) \geqslant 1$ for temperatures of $10^{6} \mathrm{~K}$ or less (for electron perturbers), we see that the overlap condition, $R_{0}>\rho_{0}$, is violated only during a strong collision. Thus, for neutral radia- 
tors, the wave functions will overlap only during a strong collision. In the case where a radiating ion accelerates a charged perturber the deBroglie wavelength of the perturber is decreased, $X_{0}=\hbar / m v_{0} \leq X_{\mathrm{av}}$. If $v_{0}$ is not much larger than $v_{\mathrm{av}}$, there is essentially no difference between this case and the neutral radiator discussed above. If $v_{0} \gg v_{\text {av }}$ the situation is somewhat different. In this case the perturber will come very close to the radiator and it is necessary to construct the initial wave packet with a width $\Delta x$ much smaller than $\chi_{\mathrm{av}}$. Since $X_{0} \ll X_{\text {av }}$, we construct the wave packet so that $\Delta x>\chi_{0}$ which, using $\Delta x \Delta p \gtrsim \hbar$, implies that $\Delta p<p_{0}$. Such a wave packet will not hold together very long, however, it will hold together for a time on the order of $\tau=\rho_{0} / v_{0}$ which is all that is required by eq (73). In this case we will have $X_{0} \ll R_{0}$ and the wave functions will overlap when $\rho_{0} \lesssim R_{0}$. The perturbation potential will be

$$
V \simeq Z e^{2} R_{0} / \rho_{0}^{2} \text { and }(V \tau / \hbar)=\left(Z e^{2} R_{0} / \hbar \rho_{0} v_{0}\right) ;
$$

we do not need to consider a $V_{\text {eff }}$ as discussed in section 7.3 because when $\rho_{0} \leq R_{0}, V$ is comparable to the ionization energy for ions [29]. If $z_{0} \gg v_{\text {av }}$ then, from eq $(70)$, we have $w_{0} \simeq\left(2 Z e^{2} / m \rho_{0}\right)^{1 / 2}$ and

$$
(V \tau / \hbar) \simeq\left[(Z / 2)\left(R_{0}^{2} / \rho_{0} a_{0}\right)\right]^{1 / 2}
$$

where $a_{0}=\hbar^{2} / m e^{2}$ is the Bohr radius (we have chosen $m$ as the electron mass; for an ion perturber $(V \tau / \hbar)$ would be even larger). Since $R_{0}$ is larger than $a_{0}$, we see that the overlap of wave functions again produces a strong collision.

It is interesting to note that, for Stark broadening in plasmas we have $(V \tau / \hbar) \simeq\left(Z e^{2} R_{0} / \hbar \rho_{0} v_{0}\right) \geq\left(Z R_{0} / a_{0} l\right)$, where $l=m \rho_{0} v_{0} / \hbar$ is the angular momentum of the perturber. Since $R_{0}>a_{0}$, we will have strong collisions when $l \lesssim 1$. Other interaction potentials may be treated in a similar manner with the same general results. In any case, the wave functions will not overlap if $\chi_{0}<\rho_{0}$; using $\chi_{0}=\hbar / m v_{0}$ and $l=m \rho_{0} v_{0} / \hbar$, we find that the radiator and perturber will be distinct (i.e., their wave functions do not overlap) when

$$
l>1 \text {. }
$$

It is also interesting to note that the perturbers will look like classical particles as they pass the radiator only if $\Delta x \ll x$ and $\Delta p \ll p$; using $\Delta x \Delta p \simeq \hbar$, we again obtain the inequality $\hbar<m \rho_{0} v_{0}$ or $l>1$. We note further that $\Delta l= \pm 1$ in a dipole transition, thus $\Delta l<l$ and it is in this sense that the angular momentum of a perturber is conserved during a collision, as it must be if we are to use classical mechanics to describe the perturbers.

We have now shown that the validity criteria for the use of classical wavepackets are all easily satisfied if $\Delta p \ll p$, or equivalently, $\Delta V \ll p^{2} / 2 m$. That is, the essential validity criterion for the classical wavepackets is the same as the condition for validity of a WKB calculation. This was also noted by Baranger [4] who found that the results of a quantum mechanical impact theory reduce to the classical path results when a WKB treatment of the wave functions is employed.

To show that the condition $\Delta V<p^{2} / 2 m$ is satisfied we note that $V$ increases monotonically as the radiatorperturber separation is decreased, thus $\Delta V \leqslant V$ and it is sufficient to show that $V<p^{2} / 2 m$. Again $V$ may be replaced by $V_{\text {eff }}$ if $V$ is not strong enough to mix adjacent states. Using $p^{2} / 2 m=m v_{0}^{2} / 2, \tau=\rho_{0} / v_{0}$, and $l=m \rho_{0} v_{0} / \hbar$, we obtain

$$
(V \tau / \hbar l)<1 .
$$

Noting eqs (68) and (76), it is clear that this inequality can be violated only during a strong collision (recall that $l \lesssim 1$ produces a strong collision).

Equation (73) requires that a perturber be represented by a wave packet when it collides with the radiator. If we let $\varphi_{\text {w.p. }}^{(i)}\left(\mathbf{x}_{j}\right)$ denote a Gaussian wave packet for the $j$ th particle then the wave function $\varphi^{(i)}\left(\mathbf{x}_{j}, t\right)=T_{j}(t) \varphi^{(i)}\left(\mathbf{x}_{j}, 0\right)$, where $T_{j}$ is the time development operator for this particle, will be a Gaussian packet at some time $t_{j}$ if we construct $\varphi^{(i)}\left(\mathbf{x}_{j}, 0\right)$ such that

$\varphi^{(i)}\left(\mathbf{x}_{j}, 0\right)=T_{j}\left(-t_{j}\right) \varphi_{\text {w.p. }}^{(i)}\left(\mathbf{x}_{j}\right)$ (note that $\left.T_{j}\left(t_{j}\right) T_{j}\left(-t_{j}\right)=1\right)$.

In this manner we can arrange the wave function $\varphi^{(i)}(\mathbf{x}, t)=\prod_{j} \varphi^{(i)}\left(\mathbf{x}_{j}, t\right)$ in such a way that all particles which collide with the radiator during the time of interest are represented by Gaussian wavepackets at the time of their collision. In general these particles will not be represented by wavepackets before or after their collisions, but this does not matter as far as eq (73) is concerned.

We have now shown that it is possible to represent the perturbers by. classical wavepackets as required by eq (73) unless a strong collision occurs (in which case we do not use wavepackets). It is interesting to note that it may be possible to treat some strong collisions by classical path methods. That is, eq (77) could be satisfied when $(V \tau / \hbar) \gtrsim 1$ if $l$ is sufficiently large. If one wished to do this, it would be necessary to reexamine the validity criteria in more detail. For the purposes of this paper, however, it is sufficient to state that the use of classical wavepackets is justified for all collisions which are not strong collisions.

\subsection{An Approximate Classical Potential}

Although we have justified the use of a classical potential as given by eq (73), we have not yet specified its functional form. If back reaction effects in $\varphi^{(i)}$ are negligible, the potential $V^{(i)}$ will result from a perturber passing by the radiator on a straight line trajectory (or a hyperbola if both radiator and perturber are charged particles). If back reaction effects are important, the trajectory will be a rather complicated function of velocity, acceleration, etc. A wavepacket formed by WKB calculations (the iterative procedure discussed in sec. 5) may be 
able to account for the true classical trajectory, however, the perturber wave function $\varphi^{(i)}$ would no longer be an $H_{p}$ eigenfunction. While this may not matter for some scattering problems, it makes a great deal of difference in line broadening.

In section 6 we used the fact that $\varphi^{(i)}$ is an $H_{p}$ eigenfunction to obtain the classical energy expression $E_{p}^{(i)}\left(\mathbf{x}^{(i)}, \mathbf{v}^{(i)}\right)$ in eq (44). If the perturber wave function were not an energy eigenfunction, the perturber density matrix would not be "diagonal in wave packet states" and the average over perturber states, eq (4l), would be intractable in a wave packet representation. We must therefore show: (1) that $\varphi^{(i)}$ may be an $H_{p}$ eigenfunction (i.e., back reaction effects in $\varphi^{(i)}$ are negligible), and (2) that the energy eigenvalues may be represented by $E_{p}^{(i)}\left(\mathbf{x}^{(i)}, \mathbf{v}^{(i)}\right)$ as stated in eq (44).

Since energy eigenvalues are not time dependent, we need only show that $E_{p}^{(i)}$ represents the $H_{p}$ eigenvalue at some instant of time. This problem is essentially the same as eq (73) except that all perturbers must be represented by classical wave packets at some instant. Since we have a large number of perturbers in our system (a cell), there will always be some wave functions which overlap; however, for virtually all problems of interest we have $X_{\text {av }} \ll n^{-1 / 3}$ and it is possible to construct wave packets for the overwhelming majority of perturbers at some instant of time (cf. eq (75)). It is in this sense that eq (44) is satisfied.

To show that back reaction effects in $\varphi^{(i)}$ are negligible, we consider the perturbation $V^{(i)}\left(\mathbf{x}^{(i)}, \mathbf{v}^{(i)}, t\right)$ which results when a perturber moves past a neutral radiator on a straight line trajectory (the trajectory will be a hyperbola if both perturber and radiator are charged particles). The true perturber trajectory will deviate from a straight line (or hyperbola) because of accelerations (back reaction) resulting from the potential $V$ seen by the perturbers (this potential may be $V_{\text {eff }}$ as discussed in sec. 7.3). The approximate trajectory will be valid if this deviation does not appreciably alter the potential $V^{(i)}$ seen by the radiator during the collision (i.e., during the time $\tau \simeq \rho_{0} / v_{0}$, or the time of interest $1 / \Delta \omega$, if this should be shorter). $V^{(i)}$ is influenced by the perturber trajectory through its dependence on $\mathbf{r}(t)$, the position of the perturber at the time $t$. If the deviation, $\Delta \mathbf{r}(t)$, produced by the perturbation is small enough that $\mathbf{r}(t)+\Delta \mathbf{r}(t) \simeq \mathbf{r}(t)$, the approximate trajectory will provide a good estimate of the true perturbation potential (whether the radiator is an ion or a neutral).

For a time on the order of $\tau$, the magnitude of the deviation may be estimated by $\Delta r=|\mathbf{F}| \tau^{2} / 2 m$ $=|\nabla V| \tau^{2} / m$ where $\mathbf{F}=-\nabla V$ is the back reaction force which causes the perturber to deviate from the approximate trajectory. Since $V$ is inversely proportional to some power of $r(t)$ we have $|\nabla V| \simeq V \mid r(t)$. Using $r(t) \geqslant \rho_{0}$ we have

$$
\frac{\Delta r}{r} \leqslant \frac{V \tau^{2}}{2 m \rho_{0}^{2}}<\frac{V \tau}{\hbar l} \ll \frac{V \tau}{\hbar}
$$

where $\tau=\rho_{0} / V_{0}, l=m \rho_{0} v_{0} / \hbar$, and $l>1$. This result shows that $(\Delta r / r) \ll 1$ unless a strong collision occurs.

The above argument shows that the approximate trajectory is valid (unless a strong collision occurs) for elastic collisions. If an inelastic collision should occur, the radiator and perturber will exchange an amount of energy $\Delta E$. This will produce a sudden change in the perturber velocity, $\Delta E=m v \Delta v$, which will give rise to a deviation $\Delta r \leqslant \tau \Delta v$ in the trajectory. Again using $\tau_{0}=\rho_{0} / v_{0}, l=m v_{0} \rho_{0} / \hbar$, and $l>1$, we obtain

$$
\frac{\Delta r}{r} \leqslant \frac{\Delta v}{v_{0}} \simeq \frac{\Delta E}{\frac{1}{2} m v_{0}^{2}} \simeq \frac{\tau \Delta E}{\hbar l}<\frac{\tau \Delta E}{\hbar} .
$$

In section 7.2 it was shown that the probability for inelastic transitions is essentially zero unless $(\tau \Delta E / \hbar) \lesssim 1$ (for a more detailed treatment see ref. [30]). Thus the approximate trajectory is valid for both elastic and inelastic collisions.

It is interesting to note, that the change in the perturbers kinetic energy during the collision is a small fraction of its total kinetic energy.

\section{Summary and Comments}

It has been found that if the approximations which are used in the classical path treatment of line broadening break down, then a strong collision, defined by eq (68), is occuring. It should be noted that the converse of this statement is not necessarily true; that is, it may be possible to extend the classical path treatment of some problems into the region where $(V \tau / \hbar) \simeq 1$. In this case the validity conditions must be considered in more detail.

In the application of classical path methods, strong collisions are usually treated by interruption approximations based on the Lorentz-Weisskopf theory; the influence of all other collisions is evaluated by means of the time development operator $T_{a}^{(i)}(t)$ as discussed in section 6 .

In section 8.4 it was shown that back reaction effects on the perturber trajectory are negligible whenever a classical path calculation is justified. That is, the validity criteria for the use of classical wave packets break down at the same time as (or in some cases before) the validity conditions for the use of unperturbed trajectories.

It was noted that our definition of strong collisions, eq (68), is essentially the same as the result obtained by setting the lead terms in an $S$-matrix expansion to unity. However, using this $S$-matrix expansion until the lead terms are on the order of unity may be a very poor approximation of the $S$-matrix; indeed, most errors attributed to strong collisions stem from this series approximation. To avoid possible confusion, we wish to emphasize that this approximation concerning the $S$-matrix is not part of the classical path approximation and any errors resulting from it have no bearing on the validity of classical path methods. It is therefore quite possible to improve upon these so-called strong collision problems within the framework of the classical path approach. The series approximation has been 
improved for Lyman alpha lines [31], by summing terms in the series and, for hydrogen lines in general [32], by an approximate treatment of the exponential definition of the $S$-matrix.

The region of validity for the weak coupling density matrix has been given as $\Delta \omega<k T / \hbar$. We wish to emphasize once again that the approximations relevant to this density matrix are not influenced by the semiclassical treatment of the wave functions which is a part of the classical path approximation. The weak coupling density matrix may be (and has been $[3,4]$ ) used in more rigorous quantum mechanical theories.

\section{References and Notes}

[11 Smith, E. W., Vidal, C. R., and Cooper, J., J. Res. Nat. Bur. Stand. (U.S.), 73A (4), 405-420 (1969).

[2] Schiff, L. I., Quantum Mechanics, p. 261. (McGraw-Hill Book Co., Inc., New York, 1955).

[3] Smith, E. W., and Hooper, C. F., Jr., Phys. Rev. 157, 126 (1967).

[4] Baranger, M., Atomic and Molecular Processes, chap. 13, edited by D. Bates (Academic Press Inc., New York, 1962).

[5] Baranger, M, Rand Corporation Report No. (RM-2118-AEC) ASTIA Document No. (AD 150692, Feb. 1958.

[6] Griem, H. R., Plasma Spectroscopy, chap. 4. (McGraw-Hill Book Co., Inc., New York, 1964).

[7] $T(t)$ governs the time development of the system from any arbitrary time $\tau$ to the time $t+\tau$; this operator may also be written $T(t+\tau, \tau)$.

[8] Fano, U., Phys. Rev. 131,259 (1963).

[9] Fano, U., Rev Mod. Phys. 29, 74 (1957).

[10] If the radiator is an ion in a plasma, the Coulomb interaction $V_{0}$, between this ion and the charged perturbers may be added to $H_{p}$ because it does not depend on the internal structure of the radiator.

[11] There are many different ways of treating ensembles; for a more detailed discussion of the approach which we use, the reader is referred to references [9] and [12]. In reference [12], the emphasis is placed on microcanonical ensembles, for which energy is one of the fixed macroscopic constraints; the results for a canonical ensemble, which we use, are stated in section (3 4) of that reference. Reference [9] gives a very good discussion of ensembles in general.

[12] Mori, H., Oppenheim, I., and Ross, J., Studies in Statistical Mechanics, pp. 240-255, edited by J. DeBoer and G. E. Uhlenbeck (North-Holland Publishing Co., Amsterdam, 1962).
[13] It is not actually necessary that the $\varphi^{(i)}$ be energy eigenfunctions. It is necessary only that each $\varphi^{(i)}$ be an eigenfunction of some complete set of commuting operators (i.e., the $\varphi^{(i)}$ are pure states). Since there may be more than one complete set of operators, there may also be more than one set of pure states. For our purposes, it is convenient to use some set of operators which contains the Hamiltonian, $H_{p}$. Results obtained with other sets will be identical with ours, but are probably more complicated.

[14] Griem, H. R., Phys. Rev 165, 258 (1967), footnote 17.

[15] An extensive study of relaxation functions of the autocorrelation type has been made by Kubo, R., J. Math. Phys. 4, 175 (1963), and Lectures in Theoretical Physics, edited by W. E. Downs and J. Down (Interscience Publisher, Inc., New York, 1959), Vol 1, p. 120. Applications to spectral line broadening in gases are discussed by Kubo in Fluctuation, Relaxation and Resonance in Magnetic Systems, edited by D. ter Haar (Plenum Press, New York, 1961), p. 49, and in sec. 2.c of Ref. 3.

[16] Margenau, H., and Lewis, M., Rev. Mod. Phys. 31, 269 (1959).

[17] Cooper, J., Rep. Progr. Phys. (London: Institute of Physics and Physical Society) 29,35 (1966), sec. (11.3).

[18] Burshtein, A. I., and Naberukhin, Yu. I., Soviet Physics JETP (English trans.) 25, 799 (1967).

[19] Schweber, S., An Introduction to Relativistic Quantum Field Theory, pp 330-334 (Harper and Row Publishers Inc., New York, 1962).

[20] Cooper, J., Phys. Rev. Letters 17, 991 (1966).

[21] Griem, H. R., Kolb, A. C., and Shen, K. Y., Phys. Rev. 116, 4 (1959).

[22] For various types of interactions one obtains different estimates of the impact parameter which gives rise to $V \gtrsim k T$; however the thermal wavelength estimate is within an order of magnitude for most problems of interest.

[23] Baranger, M., Phys. Rev. 112, 855 (1958).

[24] Messiah, A., Quantum Mechanics, Vol. 1, p. 66 (John Wiley \& Sons, Inc., New York, 1961).

[25] op. cit. ch. VI.

[26] Dirac, P. A. M., Principles of Quantum Mechanics, pp. 121-125 (Claredon Press, Oxford, 1958).

[27] Mott, N. F., and Massey, H. S. W., The Theory of Atomic Collisions p. 332 and p. 350 (Claredon Press, Oxford, 1965).

[28] Merzbacher, E., Quantum Mechanics (John Wiley \& Sons, Inc., New York, 1961).

[29] For hydrogenic ions the level spacing is on the order of $e^{2} Z^{2} / a_{0} n^{3}$ and the ionization energy is $\left(e^{2} Z^{2} / 2 n^{2} a_{0}\right)$.

[30] Seaton, M. J., Proc. Phys. Soc. 79, 1105 (1962).

[31] Griem, H. R., Phys. Rev. 140, Al140 (1965).

[32] Shen, K. Y., and Cooper, J., Ap. J. 155, 37 (1969).

(Paper 73A4-558) 